\title{
Tmem119-EGFP and Tmem119-CreERT2 Transgenic Mice for Labeling and Manipulating Microglia
}

\author{
Tobias Kaiser, ${ }^{1,2}$ and ${ }^{-}$Guoping Feng ${ }^{1,2,3}$
}

https://doi.org/10.1523/ENEURO.0448-18.2019

${ }^{1}$ McGovern Institute for Brain Research, Massachusetts Institute of Technology, Cambridge, Massachusetts 02139 , ${ }^{2}$ Department of Brain and Cognitive Sciences, Massachusetts Institute of Technology, Cambridge, Massachusetts 02139, and ${ }^{3}$ Stanley Center for Psychiatric Research, Broad Institute of MIT and Harvard, Cambridge, Massachusetts 02142

\section{Visual Abstract}

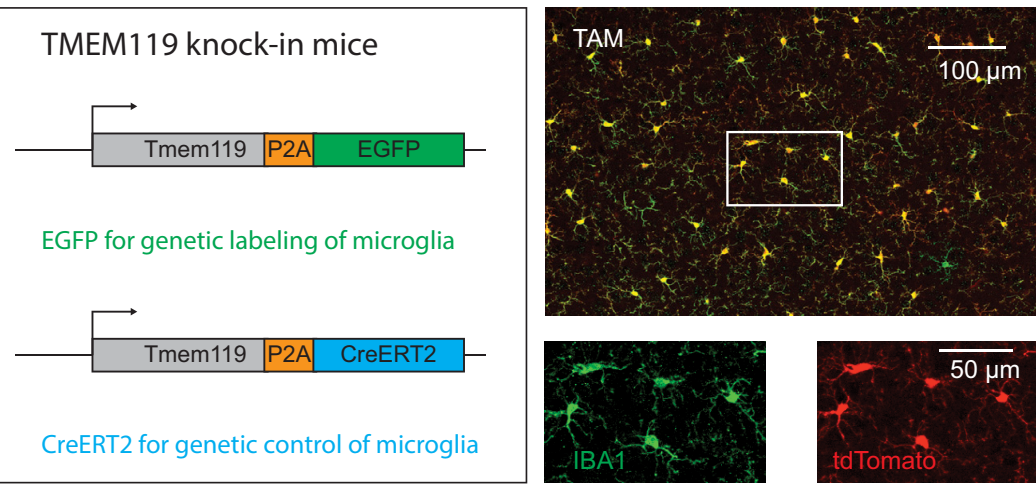

Microglia are specialized brain-resident macrophages with important functions in health and disease. To improve our understanding of these cells, the research community needs genetic tools to identify and control them in a manner that distinguishes them from closely related cell types. We have targeted the recently discovered microglia-specific Tmem119 gene to generate knock-in mice expressing EGFP (JAX\#031823) or CreERT2 (JAX\#031820) for the identification and manipulation of microglia, respectively. Genetic characterization of the locus and qPCR-based analysis demonstrate correct positioning of the transgenes and intact expression of endogenous Tmem119 in the knock-in mouse models. Immunofluorescence analysis further shows that parenchymal microglia, but not other brain macrophages, are completely and faithfully labeled in the EGFP-line at different time points of development. Flow cytometry indicates highly selective expression of EGFP in CD11 $\mathrm{b}^{+} \mathrm{CD} 45 \mathrm{lo}$ microglia. Similarly, immunofluorescence and flow cytometry analyses using a Cre-dependent reporter mouse line demonstrate activity of CreERT2 primarily in microglia upon tamoxifen administration with the

\section{Significance Statement}

Tools that specifically label and manipulate only microglia are currently unavailable, but are critically needed to further our understanding of this cell type. Complementing and significantly extending recently introduced microglia-specific immunostaining methods that have quickly become a new standard in the field, we generated two mouse lines that label and control gene expression in microglia with high specificity and made them publicly available. Using these readily accessible mice, the research community will be able to study microglia biology with improved specificity. 
caveat of activity in leptomeningeal cells. Finally, flow cytometric analyses reveal absence of EGFP expression and minimal activity of CreERT2 in blood monocytes of the Tmem119-EGFP and Tmem119-CreERT2 lines, respectively. These new transgenic lines extend the microglia toolbox by providing the currently most specific genetic labeling and control over these cells in the myeloid compartment of mice.

Key words: CreERT2; EGFP; macrophage; microglia; Tmem119; transgenic

\section{Introduction}

Microglia are specialized brain-resident macrophages that comprise $5-12 \%$ of the glial cells in the adult mouse brain (Lawson et al., 1990). Under physiologic conditions, phagocytic and secretory activity of these glia support neurogenesis, development of neuronal connectivity, and survival of neurons (Stevens et al., 2007; Sierra et al., 2010; Paolicelli et al., 2011; Schafer et al., 2012; Ueno et al., 2013; Weinhard et al., 2018). Complementing these homeostatic functions, microglia react to perturbations, which has been shown in the context of vascular injury, multiple sclerosis lesions, and neurodegeneration (Itagaki et al., 1989; Davalos et al., 2005; Ransohoff, 2016; Aguzzi and Zhu, 2017; Keren-Shaul et al., 2017; Mathys et al., 2017; O'Loughlin et al., 2018). In the future, many of these processes and their potential for therapeutic targeting will be further examined, and understanding of both homeostatic and disease-related contributions of microglia will critically depend on means to specifically identify and control them. Several mouse lines are currently available for either fluorescent labeling or Cre-expression that harness the loci of putative microglia signature genes Tie2, Runx1, Csf1r, Aif1, Lyz2, Itgam, Sall1, or CX3cr1 (Clausen et al., 1999; Sasmono et al., 2003; Ferron and Vacher, 2005; Hirasawa et al., 2005; Samokhvalov et al., 2007; Ginhoux et al., 2010; Parkhurst et al., 2013; Yona et al., 2013; Buttgereit et al., 2016). Collectively, these mouse lines have been instrumental in gaining insights on microglia. However, furthering our understanding of microglia using the currently available lines is complicated by the fact that it is difficult to distinguish microglia from other closely related peripheral and central cell types such as blood monocytes as well as perivascular, choroid plexus,

Received November 14, 2018; accepted July 20, 2019; First published August 1, 2019.

The authors declare no competing financial interests.

Author contributions: T.K. and G.F. designed research; T.K. performed research; T.K. analyzed data; T.K. and G.F. wrote the paper.

This work was supported by the Tan-Yang center for Autism Research at MIT to T.K.; by the National Institute of Mental Health (5R01MH097104), the Poitras Center for Affective Disorders Research at MIT, Tan-Yang center for Autism Research at MIT, Stanley Center for Psychiatric Research at Broad Institute of MIT and Harvard, Nancy Lurie Marks Family Foundation, Simons Foundation Autism Research Initiative (SFARI) and Simons Center for the Social Brain at MIT to G.F.; and partially supported by Cancer Center Support (core) Grant P30-CA14051 from the NCl. We thank all members of the Feng laboratory for helpful discussions, and Bailey Clear, Nicholas Sanders, Ohyoon Kwon, and Dongqing Wang for excellent technical support.

Correspondence should be addressed to Guoping Feng at Fengg@mit.edu. https://doi.org/10.1523/ENEURO.0448-18.2019

Copyright (C) 2019 Kaiser and Feng

This is an open-access article distributed under the terms of the Creative Commons Attribution 4.0 International license, which permits unrestricted use, distribution and reproduction in any medium provided that the original work is properly attributed. and meningeal macrophages (Wieghofer and Prinz, 2016; Haimon et al., 2018).

Recent advances in RNA sequencing and other cell profiling technologies have enabled the discovery of celltype-specific signature genes (Butovsky et al., 2014). Among these, transmembrane protein 119 (Tmem119) is highly and exclusively expressed in microglia in the brains of mice and humans (Bennett et al., 2016; Satoh et al., 2016). Although TMEM119-specific antibodies are already widely used as a gold standard for immunohistochemical methods, faithful fluorescent reporter lines and inducible Cre-lines that allow in vivo observation and manipulation are currently not available. Here, we report the generation and characterization of knock-in mouse lines Tmem119-EGFP (JAX\#031823) and Tmem119CreERT2 (JAX\#031820), where microglia express EGFP and CreERT2, respectively, while preserving endogenous Tmem119 expression. We demonstrate that EGFP is expressed throughout the brain and that the tag is confined to microglia only, without significantly labeling other brain macrophages. We further provide evidence that the inducible Cre is primarily active in microglia by crossing to the conditional fluorescent reporter mouse line Ai14. In these mice, we also observe activity in leptomeningeal cells that line the surface of the brain and penetrate deep into the brain ensheathing some large blood vessels. Finally, we demonstrate minimal to absent transgene expression in monocytes of the mice. These publicly available mouse lines provide valuable tools for the functional study of bona fide microglia.

\section{Materials and Methods}

\section{Animal work}

All animal procedures were performed in accordance with MIT's animal care regulations. Overall, $>30$ animals of $>10$ litters were generated per line and mice were crossed to C57BL/6J up to generation N3. For all experiments, at least three independent mice were analyzed, which included both sexes and no apparent sex differences were observed.

\section{Generation of transgenic animals using CRISPR/Cas9}

To generate Tmem119 knock-in mice, donor DNA templates encoding ribosome-skipping peptide porcine teschovirus-1 polyprotein and EGFP (P2A-EGFP) or CreERT2 (P2A-CreERT2) were synthesized. These sequences were flanked by sequences of 55-300 bp for EGFP and $1.5 \mathrm{~kb}$ for CreERT2 homologous to $5^{\prime}$ and $3^{\prime}$ regions around the Tmem119 stop codon. These templates were injected into fertilized mouse oocytes together with a single crRNA (AGUCUCCCCCAGUGUCUAAC, Synthego) that cuts at the stop codon.

Donor DNA templates were generated by digesting pAAV-P2A-EGFP (sequence below) with Xbal and EcoRl 
(New England Biolabs) and inserting three gblocks (LHA, P2A-EGFP or P2A-CreERT2, RHA, purchased from IDT, sequences below) using Gibson cloning (HIFI assembly mix, New England Biolabs) according to the manufacturers' protocols. Resulting plasmids were purified and sequenced. For CreERT2, the highly purified dsDNA plasmid was directly used as donor DNA in injections. For EGFP, single strand DNA (ssDNA) was produced using PCR with forward primers for left homology arms between 55 and 300 bp (5'-A*G*C*AACTGGTCCTCCTGAAA-3' and $5^{\prime}-$ CAAAGCCTGTGAAGGGTGGG-3', respectively; * denoting phosphorothioate) and a reverse primer for a $55 \mathrm{bp}$ right homology arm (5'-CAAAGAGGTGACCCTCAAGG$3^{\prime}$, with $5^{\prime}$ phosphorylation for lambda digest of antisense strand). Using these primers, large scale PCR with Takara PrimeStar was performed to obtain $40-60 \mu \mathrm{g}$ of product. The PCR product was highly purified with the Qiagen PCR purification kit and subject to digestion of the antisense strand. Lambda exonuclease (New England Biolabs) was used to digest $20 \mu \mathrm{g}$ of dsDNA at $37^{\circ} \mathrm{C}$ for $60 \mathrm{~min}$. Complete digestion of dsDNA was confirmed by agarose gel electrophoresis and Sanger sequencing with senseand antisense-binding primers.

Mixtures for injection of zygotes were prepared freshly on the morning of the day of injection. Briefly, water to a final volume of $100 \mu \mathrm{l}$ was mixed with final concentrations of $10 \mathrm{~mm}$ TrisHcl buffer, $0.61 \mu \mathrm{m}$ crRNA, $0.61 \mu \mathrm{m}$ tracrRNA (sequence proprietary to Synthego) and heated to $95^{\circ} \mathrm{C}$ for $5 \mathrm{~min}$. Heated mixtures were cooled to room temperature for $10 \mathrm{~min}$ and $30 \mathrm{ng} / \mu \mathrm{l}$ EnGen Cas9 protein (New England Biolabs) was added. Mixtures were incubated at $37^{\circ} \mathrm{C}$ for 15 min to form Cas9 to crRNA-tracrRNA complexes. Final concentrations of $5 \mathrm{ng} / \mu \mathrm{l}$ donor DNA (P2AEGFP ssDNA or P2A-CreERT2 plasmid dsDNA) and 10 $\mathrm{ng} / \mu \mathrm{l}$ recombinant RAD51 protein were added. Mixtures were kept on ice until use, when they were incubated at $37^{\circ} \mathrm{C}$ for $15 \mathrm{~min}$ followed by centrifugation at $10,000 \mathrm{rpm}$ for 1 min to prevent clogging of the micropipette.

Injection of mixtures was conducted using standardized protocols of the transgenics facility in zygotes obtained from pure C57BL/B6N mice (Taconic).

\section{Genetic analysis and genotyping of Tmem119 and Ai14 mice}

Founder mice were genetically examined by amplifying sequences spanning the $5^{\prime}$ and $3^{\prime}$ junction and including the entire inserted transgene. Specifically, high-quality DNA was obtained from ear punches using a tissue DNA extraction kit according to the manufacturer's instructions (Macherey-Nagel). One amplicon spanning $\sim 1.5 \mathrm{~kb}$ (Fig. 1 , open arrows) of these sequences was generated using Q5 polymerase with primers 5' of LHA-F (GCCTCTGTCACTTAAGTTGG) and P2A-R (GCTTCAGCAGGCTGAAGTTA). A second amplicon of $\sim 2.5-3.5 \mathrm{~kb}$ (Fig. 1, closed arrows) was generated using primers Tmem-LHAP2A-F (CAGTGTCGGAAGCGGAGCTA) and 3'ofRHA-R (GAAAGAGGAAGCTAGAAGGG). Both amplicons were purified and then sequenced using primers spanning the entire length using Sanger sequencing (GENEWIZ). Trace files were aligned to in silico assemblies and analyzed using Snapgene.

To genotype mice, ear tissue was prepared using an alkaline buffer ( $25 \mathrm{~mm} \mathrm{NaOH}, 0.2 \mathrm{~mm} \mathrm{Na}_{2}$ EDTA, pH 12) at $95^{\circ} \mathrm{C}$ for $30 \mathrm{~min}$. The lysed tissue solution was neutralized using an acidic buffer (40 mM TrisHcl, pH 5). PCR using primers WT-F GTCAGGAGGAGGCCCAGGAA, EGFP-F CTGCTGCCCGACAACCACTA, CreERT2-F ACCGCCTACATGCGCCCACT, and Common-R GTTTCCTGGGGTGCACCAGA yielded products of 400 bp for the wild-type allele and $320 \mathrm{bp}$ for the EGFP or CreERT2 allele.

Ai14(RCL-tdT) mice were obtained from JAX (stock 007914) and maintained in house. Homozygous Ai14 mice were bred to heterozygous Tmem119-CreERT2 mice to generate offspring for the analysis. Ai14 mice were genotyped according to the protocol provided by JAX.

\section{RNA isolation and quantitative PCR}

Brain hemispheres from adult Tmem119-EGFP ${ }^{+/-}$mice were dissected and snap-frozen in liquid nitrogen. Highly pure RNA was isolated using an RNeasy purification kit (Qiagen) following the manufacturer's instructions. Equal amounts of RNA (1.5 $\mu \mathrm{g}$ per sample) were reverse transcribed using an iScript Advanced cDNA synthesis kit (BioRad). Resulting CDNA was diluted 1:50 in ultrapure water. Quantitative PCR (qPCR) was conducted using SsOAdvanced Universal SYBR Green Supermix (Bio-Rad) on a CFX96 real-time system. Primers used were exon-spanning whenever possible and of the following sequences: Tmem119-F CCTTCACCCAGAGCTGGTTC, Tmem119-R GGCTACATCCTCCAGGAAGG, GAPDH-F GCCTTCCGTGTTCCTACC, GAPDH-R CCTCAGTGTAGCCCAAGATG, b-actin-F CTAAGGCCAACCGTGAAAAG, b-actin-R ACCAGAGGCATACAGGGACA. Differential gene expression analysis was performed using built-in software for the Bio-Rad CFX96 real-time system.

\section{Tamoxifen administration}

Tamoxifen (Sigma-Aldrich, T5648) was dissolved in corn oil at $20 \mathrm{mg} / \mathrm{ml}$ under agitation for several hours in the dark and kept at room temperature for 2-3 d. Using needles from Harvard Apparatus (52-4025), separately housed adult animals were fed via oral gavage for $3 \mathrm{~d}$. Needles were rinsed between days and syringes were replaced. At time of administration, the mice weighed $\sim 19-26 \mathrm{~g}$ and received $250-400 \mu \mathrm{l}$ of the $20 \mathrm{mg} / \mathrm{ml}$ tamoxifen solution, corresponding to $0.2 \mathrm{mg} / \mathrm{g}$ body weight. Postnatal day $(\mathrm{P}) 2$ mice weighing $\sim 2 \mathrm{~g}$ received 5 $\mu \mathrm{l}$ of the $20 \mathrm{mg} / \mathrm{ml}$ solution, corresponding to $50 \mu \mathrm{g} / \mathrm{g}$ body weight per day for 3 consecutive days. The health of the mice was monitored daily. At least $7 \mathrm{~d}$ week after the last dose, mice were killed by transcardiac perfusion.

\section{Immunofluorescence staining and imaging}

Adult mice were deeply anesthetized and perfused with $25 \mathrm{ml}$ PBS followed by $25 \mathrm{ml} 4 \%$ paraformaldehyde (PFA) in PBS. Early postnatal mice (P3) were perfused with $8 \mathrm{ml}$ PBS and $8 \mathrm{ml}$ PFA. Brains were surgically removed and postfixed in the fixative at $4^{\circ} \mathrm{C}$ for $24 \mathrm{~h}$. Fixed brains were washed once in PBS and sliced into 100- $\mu \mathrm{m}$-thick sagittal slices using a Leica VT1000S. Slices were washed twice 
A

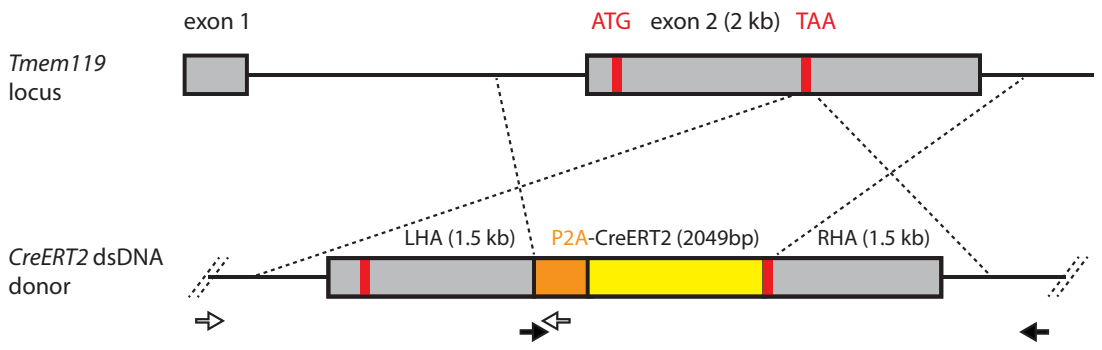

LHA (300 bp) P2A-EGFP (786 bp)

RHA (55 bp)

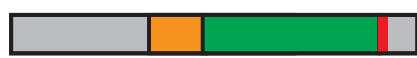

EGFP ssDNA

donor

D

CTTGGCAGGTCTTTTGACCTC

$C T T G G C A G G T C T T T T G A C C T C$

WT G G G TAG GTAGCT CT CA G GCT T

Tg G G G TAGGTAGCTCTCAGGCTT

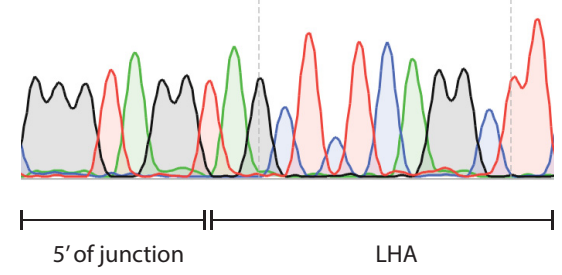

H

WT

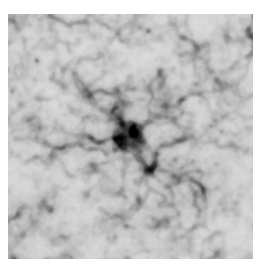

I

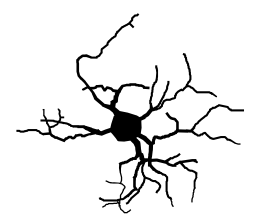

$\mathrm{EGFP}^{+/}$
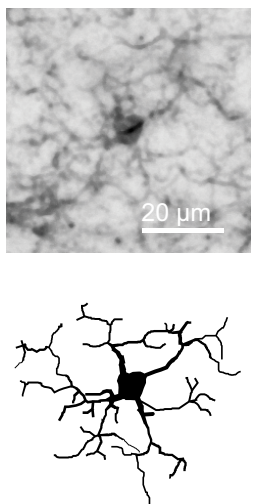

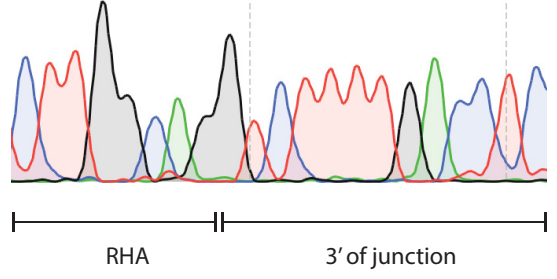

J

Cell body area $\left(\mu \mathrm{m}^{2}\right)$

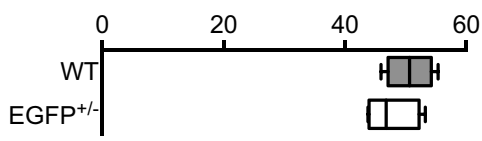

K

Number of processes

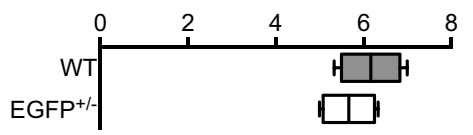

B

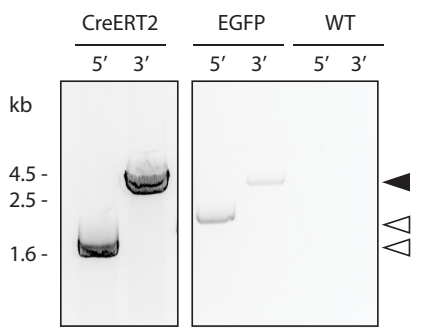

E

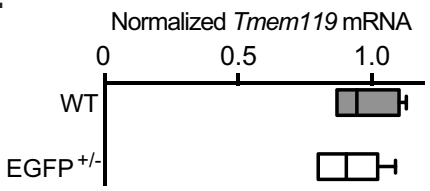

$\mathbf{F}$

WT

$\mathrm{EGFP}^{+/-}$

G
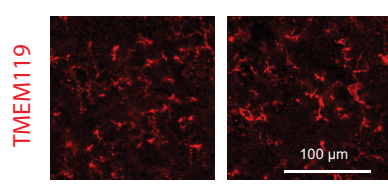

Normalized TMEM119 IF Intensity

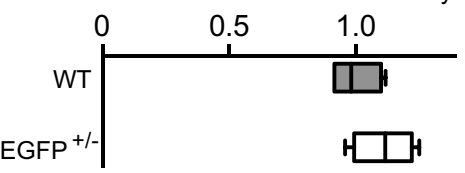

$\mathbf{L}$

Convex hull area $\left(\mu \mathrm{m}^{2}\right)$

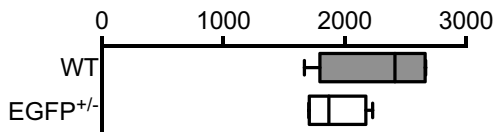

M Total process length $(\mu \mathrm{m})$

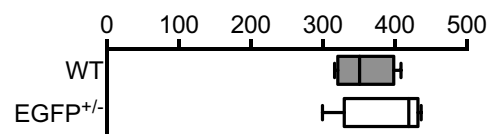

Figure 1. Generation and validation of Tmem119-EGFP and Tmem119-CreERT2 knock-in lines. A, Experimental strategy using a guide RNA (blue bar) to introduce a double-strand break at the Tmem119 stop codon in mouse zygotes and an injection mixture containing EGFP ssDNA template with short homology arms of 55-300 bp or CreERT2 dsDNA template with long $1.5 \mathrm{~kb}$ homology arms. Each knock-in is designed to cause in-frame insertion of P2A peptide followed by EGFP or CreERT2 and a stop codon. Not drawn to scale. $\boldsymbol{B}$, Confirmation of insertion by amplification of fragments spanning the $5^{\prime}$ and $3^{\prime}$ junctions using sets of primers binding inside the template and outside the homology arms $(\boldsymbol{A}$, open and closed arrows with arrowheads indicating corresponding products). C, $\boldsymbol{D}$, Sanger sequencing across the $5^{\prime}$ and $3^{\prime}$ junction of the CreERT2 line and comparison to WT sequence indicate seamless insertion in the transgenic mice (Tg). E, qPCR analysis of gene expression for Tmem119 in WT controls and Tmem119EGFP ${ }^{+/-}$knock-in animals. $N=4 \mathrm{WT}, N=6$ Tmem119-EGFP ${ }^{+/-}$mice. $\boldsymbol{F}$, Representative TMEM119 immunoreactivity in WT controls and Tmem119-EGFP ${ }^{+/-}$knock-in animals. G, Relative intensity of TMEM119 immunostaining signal. $N=4 \mathrm{WT}, \mathrm{N}=4 \mathrm{Tmem} 119-$ EGFP ${ }^{+/-}$mice. $\boldsymbol{H}, \boldsymbol{I}$, Representative IBA-immunostained microglia and corresponding Neurolucida traces in WT and Tmem119EGFP ${ }^{+/-}$knock-in mice. $\mathbf{J}-\boldsymbol{M}$, Quantified cell body area, number of processes, convex hull area, and total process length of microglia in WT and Tmem119-EGFP ${ }^{+/-}$knock-in mice. $N=12$ microglia from four mice per genotype. TAA (red bar), stop codon. 
in PBS, permeabilized in 1.2\% TX100 in PBS for $15 \mathrm{~min}$, washed twice in PBS, and subject to incubation in blocking solution ( $5 \%$ normal goat serum, $2 \%$ bovine serum albumin, $0.2 \%$ TX100 in PBS). Blocked sections were incubated with primary antibodies for IBA1 (1:500; Synaptic Systems, 234006), GFP (1:1000, Invitrogen, A11122; 1:500, Aves Labs, GFP-1020), TMEM119 (1:1000; Abcam, ab209064), Olig2 (1:1000; Millipore, AB9610), NeuN (1: 1000; Millipore, MAB377), GFAP (1:1000; Sigma-Aldrich, G9269), or CD163 (1:200; Abcam, ab182422, antigen retrieval required) for $24 \mathrm{~h}$ at $4^{\circ} \mathrm{C}$. Primary antibody incubation was followed by three washes in PBS and incubation with species-matched and Alexa fluorophore-conjugated secondary antibodies raised in goat (Invitrogen; 1:1000) for $2 \mathrm{~h}$. DAPI (1:10.000) was included in a washing step or secondary antibody incubation. Slices were washed three times in PBS and mounted and coverslipped using VECTASHIELD H-1000 mounting medium.

For immunostaining of CD163, an antigen retrieval step was included. Briefly, vibratome slices were washed twice in PBS, incubated in a retrieval buffer $(10 \mathrm{~mm}$ Sodium citrate, $\mathrm{pH}$ 8.5) for $5 \mathrm{~min}$, followed by incubation in the retrieval buffer at $80^{\circ} \mathrm{C}$ for $30 \mathrm{~min}$. Sections were cooled to room temperature and washed twice in PBS. Blocking and immunostaining were then conducted as described for other antibodies in the immunofluorescence staining and imaging methods section.

For imaging, slides were scanned on an Olympus FluoView FV1000 fixed stage confocal microscope (highpower magnifications) or Olympus BX61 epifluorescence microscope (sagittal section montage) using built-in software. For coexpression analysis in the Tmem119-EGFP line, maximum projections of four $z$-stacks from three $20 \times$ high-power fields ( $\sim 100$ cells per region) were counted manually.

For microglia morphology analysis, $z$-stacks were acquired at $20 \times$ magnification with $2 \times$ digital zoom and 1.2 $\mu \mathrm{m}$ step size. Images were imported as stacks into Neurolucida software. Cell bodies in the middle of the stacks with complete process arbors were selected and manually traced. Raw trace files were imported into Neurolucida explorer software and convex hull area, number of processes and total process length were determined using the convex hull analysis and branched structure analysis functions. Rendered traces were exported as monochrome vector graphics. Cell body areas were separately determined in FIJI using the polygon tool.

\section{Cell suspension preparation}

Microglia and blood monocytes for single-cell suspensions for flow cytometry were prepared as follows. Mice were deeply anesthetized with isoflurane and transcardially perfused with ice-cold HBSS. During perfusion, $2 \mathrm{ml}$ of whole blood was collected from the right atrium into tubes containing $40 \mu \mathrm{l}$ of $10 \%(\mathrm{w} / \mathrm{v})$ EDTA. The $2 \mathrm{ml}$ of whole blood were added to $40 \mathrm{ml}$ of red blood cell lysis buffer (Abcam, ab204733) and incubated for $10 \mathrm{~min}$ at room temperature to lyse red blood cells. The suspension containing lysed RBCs and white blood cells was spun down at $300 \times g$ for $5 \mathrm{~min}$ at $4^{\circ} \mathrm{C}$, resuspended in $10 \mathrm{ml}$ ice-cold HBSS, and pelleted again at $300 \times g$ for $5 \mathrm{~min}$ at $4^{\circ} \mathrm{C}$. WBCs were resuspended in $500 \mu \mathrm{l}$ ice-cold FACS buffer (HBSS, $0.5 \%$ BSA, $1 \mathrm{~mm}$ EDTA) and subject to staining. In parallel with the WBC enrichment, brains were rapidly dissected into $2 \mathrm{ml}$ ice-cold HBSS and cerebella and brainstem were removed. Brains were minced into small pieces and transferred to a Dounce homogenizer containing $5 \mathrm{ml}$ ice-cold HBSS with $20 \mu \mathrm{g} / \mathrm{ml}$ DNase I (Worthington, DPRF, LS006343). Tissue chunks were homogenized with 15 loose and 15 more tight strokes and the homogenate was transferred to a $50 \mathrm{ml}$ falcon tube through a pre-wet $70 \mu \mathrm{m}$ strainer. The strainer was rinsed with HBSS to top off the volume of each sample to $10 \mathrm{ml}$. Filtered homogenates were transferred to $15 \mathrm{ml}$ falcon tubes and spun at $300 \times g$ for $5 \mathrm{~min}$ at $4^{\circ} \mathrm{C}$. Supernatants were carefully removed, and pellets resuspended in $10 \mathrm{ml}$ of ice-cold $40 \%$ Percoll in HBSS. Samples were spun at $500 \times g$ for $30 \mathrm{~min}$ at $4^{\circ} \mathrm{C}$ with full acceleration and deceleration. Myelin and debris from the supernatant were carefully removed and pellets resuspended in $10 \mathrm{ml}$ ice-cold HBSS. Following another spin at $300 \times g$ for 5 min at $4^{\circ} \mathrm{C}$, the supernatant was removed and the microglial pellet resuspended in $1 \mathrm{ml}$ FACS buffer $(0.5 \%$ BSA HBSS, 1 mM EDTA).

\section{Staining for flow cytometry}

Suspensions of white blood cells and microglia were transferred to $2 \mathrm{ml}$ Eppendorf microcentrifuge tubes, and a small fraction of sample was removed for single-color controls. To stain dead cells, live/dead violet (1:500-1: 1000; ThermoFisher, L34955) was added and incubated for 5 min on ice. Tubes with live/dead-stained cells were topped off to $2 \mathrm{ml}$ with FACS buffer (0.5\% BSA HBSS, 1 mM EDTA) and spun down at $300 \times g$ for 5 min at $4^{\circ} \mathrm{C}$. Pellets were resuspended and incubated with 1:200 Mouse Fc Block (BD, 2.4G2, 553142) on ice for $15 \mathrm{~min}$. Samples were incubated with 1:200 rat anti CD45-APC/ Cy7 (BioLegend, 103115) and rat anti CD11b-PE/Cy5 (BioLegend, 101209) at $4^{\circ} \mathrm{C}$. Tubes were topped off to $2 \mathrm{ml}$ with ice-cold FACS buffer and microglia pelleted at $300 \times g$ for 5 min at $4^{\circ} \mathrm{C}$. Supernatants were removed and microglia resuspended in $500 \mu \mathrm{l}$. Resuspended microglia were filtered through corning strainer polystyrene tubes (Corning, 352235). Flow cytometry data were acquired on Aria II, Fortessa HTS, and LSRII HTS, and analyzed using FlowJo.

\section{Statistical analysis}

Quantitative data from the qPCR, immunofluorescence, and microglia morphology experiments were analyzed using GraphPad prism. Testing for normality was not possible because $N=4-6$ (4-6 independent mice were used per group). Not assuming Gaussian distribution of the data, a nonparametric test (Mann-Whitney) was used to compare median expression in both groups.

\section{DNA sequences}

PAAV-P2A-EGFP

CCTGCAGGCAGCTGCGCGCTCGCTCGCTC ACTGAGGCCGCCCGGGCAAAGCCCGGGCGTCGGGCGA CCTITGGTCGCCCGGCCTCAGTGAGCGAGCGAGCGCGC AGAGAGGGAGTGGCCAACTCCATCACTAGGGGTTCCTG 
CGGCCGCACGCGTTIAATTAAGTGTCTAGACTGCAGAG GGCCCTGCGTATGAGTGCAAGTGGGTITAGGACCAGG ATGAGGCGGGGTGGGGGTGCCTACCTGACGACCGACCC CGACCCACTGGACAAGCACCCAACCCCCATTCCCCAAA TTGCGCATCCCCTATCAGAGAGGGGGAGGGGAAACAGG ATGCGGCGAGGCGCGTGCGCACTGCCAGCTTCAGCACC GCGGACAGTGCCTTCGCCCCCGCCTGGCGGCGCGCGCC ACCGCCGCCTCAGCACTGAAGGCGCGCTGACGTCACTC GCCGGTCCCCCGCAAACTCCCCTTCCCGGCCACCTTGG TCGCGTCCGCGCCGCCGCCGGCCCAGCCGGACCGCACC ACGCGAGGCGCGAGATAGGGGGGCACGGGCGCGACCAT CTGCGCTGCGGCGCCGGCGACTCAGCGCTGCCTCAGTC TGCGGTGGGCAGCGGAGGAGTCGTGTCGTGCCTGAGAG CGCAGTCGAGAAACCGGCTAGAGGATCCTTCGAAACCG GTGCTAGCAGCGCTGTTAACGGAAGCGGAGCCACTAAC TTCTCCCTGTTGAAACAAGCAGGGGATGTCGAAGAGAA TCCCGGGCCAATGGTGAGCAAGGGCGAGGAGCTGTTCA CCGGGGTGGTGCCCATCCTGGTCGAGCTGGACGGCGAC GTAAACGGCCACAAGTTCAGCGTGTCCGGCGAGGGCGA GGGCGATGCCACCTACGGCAAGCTGACCCTGAAGTTCA TCTGCACCACCGGCAAGCTGCCCGTGCCCTGGCCCACC CTCGTGACCACCTTCGGCTACGGCCTGCAGTGCTTCGC CCGCTACCCCGACCACATGAAGCAGCACGACTTCTTCA AGTCCGCCATGCCCGAAGGCTACGTCCAGGAGCGCACC ATCTTCTTCAAGGACGACGGCAACTACAAGACCCGCGC CGAGGTGAAGTTCGAGGGCGACACCCTGGTGAACCGCA TCGAGCTGAAGGGCATCGACTTCAAGGAGGACGGCAAC ATCCTGGGGCACAAGCTGGAGTACAACTACAACAGCCA CAACGTCTATATCATGGCCGACAAGCAGAAGAACGGCA TCAAGGTGAACTTCAAGATCCGCCACAACATCGAGGAC GGCAGCGTGCAGCTCGCCGACCACTACCAGCAGAACAC CCCCATCGGCGACGGCCCCGTGCTGCTGCCCGACAACC ACTACCTGAGCTACCAGTCCGCCCTGAGCAAAGACCCC AACGAGAAGCGCGATCACATGGTCCTGCTGGAGTTCGT GACCGCCGCCGGGATCACTCTCGGCATGGACGAGCTGT ACAAGTAAGGCGCGCCCCTGCAGGGAATTCGATATCAA GCTTATCGATAATCAACCTCTGGATTACAAAATTTGTG AAAGATTGACTGGTATTCTTAACTATGTTGCTCCTTTT ACGCTATGTGGATACGCTGCTTTAATGCCTTTGTATCA TGCTATTGCTTCCCGTATGGCTTTCATTTTCTCCTCCT TGTATAAATCCTGGTTGCTGTCTCTTTATGAGGAGTTG TGGCCCGTTGTCAGGCAACGTGGCGTGGTGTGCACTGT GTTTGCTGACGCAACCCCCACTGGTTGGGGCATTGCCA CCACCTGTCAGCTCCTTTCCGGGACTTTCGCTTTCCCC CTCCCTATTGCCACGGCGGAACTCATCGCCGCCTGCCT TGCCCGCTGCTGGACAGGGGCTCGGCTGTTGGGCACTG ACAATTCCGTGGTGTTGTCGGGGAAATCATCGTCCTTT CCTTGGCTGCTCGCCTATGTTGCCACCTGGATTCTGCG CGGGACGTCCTTCTGCTACGTCCCTTCGGCCCTCAATC CAGCGGACCTTCCTTCCCGCGGCCTGCTGCCGGCTCTG CGGCCTCTTCCGCGTCTTCGCCTTCGCCCTCAGACGAG TCGGATCTCCCTITGGGCCGCCTCCCCGCATCGATACC GAGCGCTGCTCGAGAGATCTACGGGTGGCATCCCTGTG ACCCCTCCCCAGTGCCTCTCCTGGCCCTGGAAGTTGCC ACTCCAGTGCCCACCAGCCTTGTCCTAATAAAATTAAG TTGCATCATTTTGTCTGACTAGGTGTCCTTCTATAATA TTATGGGGTGGAGGGGGGTGGTATGGAGCAAGGGGCAA GTTGGGAAGACAACCTGTAGGGCCTGCGGGGTCTATTG GGAACCAAGCTGGAGTGCAGTGGCACAATCTTGGCTCA CTGCAATCTCCGCCTCCTGGGTTCAAGCGATTCTCCTG
CCTCAGCCTCCCGAGTTGTTGGGATTCCAGGCATGCAT GACCAGGCTCAGCTAATTTTTGTTTTTTTGGTAGAGAC GGGGTTTCACCATATTGGCCAGGCTGGTCTCCAACTCC TAATCTCAGGTGATCTACCCACCTTGGCCTCCCAAATT GCTGGGATTACAGGCGTGAACCACTGCTCCCTTCCCTG TCCTTCTGATITGTAGGTAACCACGTGCGGACCGAGC GGCCGCAGGAACCCCTAGTGATGGAGTTGGCCACTCCC TCTCTGCGCGCTCGCTCGCTCACTGAGGCCGGGCGACC AAAGGTCGCCCGACGCCCGGGCTITGCCCGGGCGGCCT CAGTGAGCGAGCGAGCGCGCAGCTGCCTGCAGGGGCGC CTGATGCGGTATTTTCTCCTTACGCATCTGTGCGGTAT TTCACACCGCATACGTCAAAGCAACCATAGTACGCGCC CTGTAGCGGCGCATTAAGCGCGGCGGGTGTGGTGGTTA CGCGCAGCGTGACCGCTACACTTGCCAGCGCCCTAGCG CCCGCTCCTTTCGCTTTCTTCCCTTCCTTTCTCGCCAC GTTCGCCGGCTTCCCCGTCAAGCTCTAAATCGGGGGC TCCCTTTAGGGTTCCGATTTAGTGCTTTACGGCACCTC GACCCCAAAAAACTTGATTTGGGTGATGGTTCACGTAG TGGGCCATCGCCCTGATAGACGGTTTTTCGCCCTITGA CGTTGGAGTCCACGTTCTTTAATAGTGGACTCTTGTTC CAAACTGGAACAACACTCAACCCTATCTCGGGCTATTC TTTTGATTTATAAGGGATTTTGCCGATTTCGGCCTATT GGTTAAAAAATGAGCTGATTTAACAAAAATTTAACGCG AATTTTAACAAAATATTAACGTTTACAATTTTATGGTG CACTCTCAGTACAATCTGCTCTGATGCCGCATAGTTAA GCCAGCCCCGACACCCGCCAACACCCGCTGACGCGCCC TGACGGGCTTGTCTGCTCCCGGCATCCGCTTACAGACA AGCTGTGACCGTCTCCGGGAGCTGCATGTGTCAGAGGT TाTCACCGTCATCACCGAAACGCGCGAGACGAAAGGGC CTCGTGATACGCCTATTTTTATAGGTTAATGTCATGAT AATAATGGTTTCTTAGACGTCAGGTGGCACTTTTCGGG GAAATGTGCGCGGAACCCCTATTTGTTTATTTTTTCTAA ATACATTCAAATATGTATCCGCTCATGAGACAATAACC CTGATAAATGCTTCAATAATATTGAAAAAGGAAGAGTA TGAGTATTCAACATTTCCGTGTCGCCCTTATTCCCTTT TTTGCGGCATTTTTGCCTTCCTGTTTTTTGCTCACCCAGA AACGCTGGTGAAAGTAAAAGATGCTGAAGATCAGTTGG GTGCACGAGTGGGTTACATCGAACTGGATCTCAACAGC GGTAAGATCCTTGAGAGTTTTCGCCCCGAAGAACGTIT TCCAATGATGAGCACTTTTAAAGTTCTGCTATGTGGCG CGGTATTATCCCGTATTGACGCCGGGCAAGAGCAACTC GGTCGCCGCATACACTATTCTCAGAATGACTTGGTTGA GTACTCACCAGTCACAGAAAAGCATCTTACGGATGGCA TGACAGTAAGAGAATTATGCAGTGCTGCCATAACCATG AGTGATAACACTGCGGCCAACTTACTTCTGACAACGAT CGGAGGACCGAAGGAGCTAACCGCTIIITGCACAACA TGGGGGATCATGTAACTCGCCTTGATCGTTGGGAACCG GAGCTGAATGAAGCCATACCAAACGACGAGCGTGACAC CACGATGCCTGTAGCAATGGCAACAACGTTGCGCAAAC TATTAACTGGCGAACTACTTACTCTAGCTTCCCGGCAA CAATTAATAGACTGGATGGAGGCGGATAAAGTTGCAGG ACCACTTCTGCGCTCGGCCCTTCCGGCTGGCTGGTTTA TTGCTGATAAATCTGGAGCCGGTGAGCGTGGGTCTCGC GGTATCATTGCAGCACTGGGGCCAGATGGTAAGCCCTC CCGTATCGTAGTTATCTACACGACGGGGAGTCAGGCAA CTATGGATGAACGAAATAGACAGATCGCTGAGATAGGT GCCTCACTGATTAAGCATTGGTAACTGTCAGACCAAGT TTACTCATATATACTTTAGATTGATTTAAAACTTCATT TTTAATTTAAAAGGATCTAGGTGAAGATCCTTTTTGAT AATCTCATGACCAAAATCCCTTAACGTGAGTTTTCGTT 
CCACTGAGCGTCAGACCCCGTAGAAAAGATCAAAGGAT CTTCTTGAGATCCTTTTTTTTCTGCGCGTAATCTGCTGC TTGCAAACAAAAAAACCACCGCTACCAGCGGTGGTTTG TTTGCCGGATCAAGAGCTACCAACTCTTTTTCCGAAGG TAACTGGCTTCAGCAGAGCGCAGATACCAAATACTGTC CTTCTAGTGTAGCCGTAGTTAGGCCACCACTTCAAGAA CTCTGTAGCACCGCCTACATACCTCGCTCTGCTAATCC TGTTACCAGTGGCTGCTGCCAGTGGCGATAAGTCGTGT CTTACCGGGTTGGACTCAAGACGATAGTTACCGGATAA GGCGCAGCGGTCGGGCTGAACGGGGGGTTCGTGCACAC AGCCCAGCTTGGAGCGAACGACCTACACCGAACTGAGA TACCTACAGCGTGAGCTATGAGAAAGCGCCACGCTTCC CGAAGGGAGAAAGGCGGACAGGTATCCGGTAAGCGGCA GGGTCGGAACAGGAGAGCGCACGAGGGAGCTTCCAGGG GGAAACGCCTGGTATCTTTATAGTCCTGTCGGGTTTCG CCACCTCTGACTTGAGCGTCGATTTTTGTGATGCTCGT CAGGGGGGCGGAGCCTATGGAAAAACGCCAGCAACGCG GCCTTTTTACGGTTCCTGGCCTTTTGCTGGCCTTTTGC TCACATGT

LHA

GCGGCCGCACGCGTTAATTAAGTGTCTAATTGGACAG GGAGATGGCTCAGGTGTGGAAAGCCAATATAGCGGTGC ATGCTTGCAAGGCCAGGAGTAATGATGGGGACATAGGA GGATTCTGGGAGCCTGGCCGGATAACCTATCCCCTAAC ACATAAGCACCAGGTCCCACCCAGTAAGAGGCCCTGCC TCGAAGAAGAAGTGGAGGGTCCCTGAGGAGGAAGGGCA TTTGGGGTTATCCTCTGGTCTCCAAATGCATCCCTCCT CCCACACAAACACACAAAGACACTTTCACTTGTATCTG ATGCCTGGTTAGCCATGAGCGTAAGCTAGGATTGTGAA TTCAGCCACCCTGGTACAGCAACTATTGAGTGCTTAGT GTATACTCTGGGTTCGAAACACCGAGGACAGAAATGAA TAAGATGCAGTTTCTGTCCTCAGGACCACCAGAAGGAA ACCAGGACGGGTGGCATCCTGTGTAGACAGACCCCGGA GTGTGTGCGTGTTGCTTAGCTAAGTGATTTTCTGGAGT GTGTTCTGGCTCCCCGGCCGTCCTCCCGAACTAGCAGA CCACTCGTGCTGCCGGAGTCCTGGAGCGACAGGAGACT CAGCCTGACTTCTCTCTTAACTCTCCCTCCGCGCAGGT TCCATAGCTCAACATGGTCCCCTGGTTCCTCCTGTCTC TGCTGCTACTTGCGAGGCCTGTGCCTGGGGTGGCCTAC TCTGTGTCACTCCCGGCCTCCTTCCTGGAGGATGTAGC CGGCAGCGGGGAAGCTGAGGGTTCTTCAGCCTCTTCCC CGAGCCTGCCGCCGCCTGGGACTCCAGCCTTCAGTCCC ACACCGGAGAGACCCCAGCCCACAGCTCTGGACGGCCC CGTGCCACCCACCAACCTCCTGGAAGGGATCATGGATT TCTTCCGGCAGTACGTGATGCTCATCGCGGTGGTGGGC TCGCTGACCTTCCTCATCATGTTCATAGTCTGCGCCGC CCTCATCACGCGCCAGAAGCACAAGGCCACAGCCTACT ACCCATCCTCGTTCCCTGAAAAGAAGTATGTGGACCAG AGAGACCGGGCTGGGGGACCCCGTACCTTCAGCGAGGT CCCTGACAGGGCACCTGACAGCCGGCATGAAGAAGGCC TGGACACCTCCCATCAGCTCCAGGCTGACATTCTGGCT GCTACCCAGAACCTCCGGTCTCCAGCTAGAGCCCTGCC AGGCAATGGGGAGGGAGCAAAGCCTGTGAAGGGTGGGT CGGAGGAGGAGGAGGAAGAGGTGCTCAGCGGTCAGGAG GAGGCCCAGGAAGCCCCAGTATGTGGGGTCACTGAAGA GAAGCTGGGGGTCCCAGAGGAGTCGGTCTCAGCAGAGG CTGAAGGGGTTCCTGCCACCAGTGAGGGCCAAGGGGAA GCAGAAGGGTCTTTCTCCTTAGCCCAGGAATCCCAGGG AGCAACTGGTCCTCCTGAAAGTCCCTGTGCCTGCAACA GAGTCTCTCCCAGTGTC

\section{P2A-CreERT2}

TGTGCCTGCAACAGAGTCTCTCCCAGTGTCG GAAGCGGAGCTACTAACTTCAGCCTGCTGAAGCAG GCTGGAGACGTGGAGGAGAACCCTGGACCTATGGCCAA TTIACTGACCGTACACCAAAATTTGCCTGCATTACCGGTC GATGCAACGAGTGATGAGGTTCGCAAGAACCTGATGGAC ATGTTCAGGGATCGCCAGGCGTTITCTGAGCATACCTG GAAAATGCTTCTGTCCGTTTGCCGGTCGTGGGCGGCATG GTGCAAGTTGAATAACCGGAAATGGTTTCCCGCAGAACCT GAAGATGTTCGCGATTATCTTCTATATCTTCAGGCG CGCGGTCTGGCAGTAAAAACTATCCAGCAACATTTGGGCC AGCTAAACATGCTTCATCGTCGGTCCGGGCTGCCACGAC CAAGTGACAGCAATGCTGTTTCACTGGTTATGCGGCGGAT CCGAAAAGAAAACGTTGATGCCGGTGAACGTGCAAAACA GGCTCTAGCGTTCGAACGCACTGATTTCGACCAGGTTC GTTCACTCATGGAAAATAGCGATCGCTGCCAGGATATA CGTAATCTGGCATTTCTGGGGATTGCTTATAACACCCT GTTACGTATAGCCGAAATTGCCAGGATCAGGGTTAAAG ATATCTCACGTACTGACGGTGGGAGAATGTTAATCCAT ATTGGCAGAACGAAAACGCTGGTTAGCACCGCAGGTGT AGAGAAGGCACTTAGCCTGGGGGTAACTAAACTGGTCG AGCGATGGATTTCCGTCTCTGGTGTAGCTGATGATCCG AATAACTACCTGTTTTGCCGGGTCAGAAAAAATGGTGT TGCCGCGCCATCTGCCACCAGCCAGCTATCAACTCGCG CCCTGGAAGGGATTTTTGAAGCAACTCATCGA TTGATITACGGCGCTAAGGATGACTCTGGTCAGAGATA CCTGGCCTGGTCTGGACACAGTGCCCGTGTCGGAGCCG CGCGAGATATGGCCCGCGCTGGAGTTTCAATACCGGAGA TCATGCAAGCTGGTGGCTGGACCAATGTAAATATTGT CATGAACTATATCCGTAACCTGGATAGTGAAACAGGGGCA ATGGTGCGCCTGCTGGAAGATGGCGATCTCGAGCCA TCTGCTGGAGACATGAGAGCTGCCAACCTITGGCCAAGC CCGCTCATGATCAAACGCTCTAAGAAGAACAGCCTGG CCTTGTCCCTGACGGCCGACCAGATGGTCAGTGCCTTG TTGGATGCTGAGCCCCCCATACTCTATTCCGAGTATGA TCCTACCAGACCCTTCAGTGAAGCTTCGATGATGGGCTTA CTGACCAACCTGGCAGACAGGGAGCTGGTTCACAT GATCAACTGGGCGAAGAGGGTGCCAGGCTTTGTGGATT GACCCTCCATGATCAGGTCCACCTTCTAGAATGTGCCTGG CTAGAGATCCTGATGATTGGTCTCGTCTGGCGCTCCATGG AGCACCCAGTGAAGCTACTGTTTGCTCCTAACTTGCTCTT GGACAGGAACCAGGGAAAATGTGTAGAGGGCATGGTGG AGATCTTCGACATGCTGCTGGCTACATCATCTCGGTTC CGCATGATGAATCTGCAGGGAGAGGAGTTTGTGTGCCT CAAATCTATTATTTTTGCTTAATTCTGGAGTGTACACAT TTCTGTCCAGCACCCTGAAGTCTCTGGAAGAGAAGGAC CATATCCACCGAGTCCTGGACAAGATCACAGACACTTT GATCCACCTGATGGCCAAGGCAGGCCTGACCCTGCAGC AGCAGCACCAGCGGCTGGCCCAGCTCCTCCTCATCCTC TCCCACATCAGGCACATGAGTAACAAAGGCATGGAGCA TCTGTACAGCATGAAGTGCAAGAACGTGGTGCCCCTCT ATGACCTGCTGCTGGAGGCGGCGGACGCCCACCGCCTA CATGCGCCCACTAGCCGTGGAGGGGCATCCGTGGAGGA GACGGACCAAAGCCACTTGGCCACTGCGGGCTCTACTT CATCGCATTCCTTGCAAAAGTATTACATCACGGGGGAG GCAGAGGGTTTCCCTGCCACAGCTTGACATGCCCCAGA ACTGCTGGGACCCGAATGT

$P 2 A-E G F P$

TGTGCCTGCAACAGAGTCTCTCCCAGTGTCGGAAGCG GAGCTACTAACTTCAGCCTGCTGAAGCAGGCTGGAGAC 
GTGGAGGAGAACCCTGGACCTATGGTGAGCAAGGGCGA GGAGCTGTTCACCGGGGTGGTGCCCATCCTGGTCGAGC TGGACGGCGACGTAAACGGCCACAAGTTCAGCGTGTCC GGCGAGGGCGAGGGCGATGCCACCTACGGCAAGCTGAC CCTGAAGTTCATCTGCACCACCGGCAAGCTGCCCGTGC CCTGGCCCACCCTCGTGACCACCCTGACCTACGGCGTG CAGTGCTTCAGCCGCTACCCCGACCACATGAAGCAGCA CGACTTCTTCAAGTCCGCCATGCCCGAAGGCTACGTCC AGGAGCGCACCATCTTCTTCAAGGACGACGGCAACTAC AAGACCCGCGCCGAGGTGAAGTTCGAGGGCGACACCCT GGTGAACCGCATCGAGCTGAAGGGCATCGACTTCAAGG AGGACGGCAACATCCTGGGGCACAAGCTGGAGTACAAC TACAACAGCCACAACGTCTATATCATGGCCGACAAGCA GAAGAACGGCATCAAGGTGAACTTCAAGATCCGCCACA ACATCGAGGACGGCAGCGTGCAGCTCGCCGACCACTAC CAGCAGAACACCCCCATCGGCGACGGCCCCGTGCTGCT GCCCGACAACCACTACCTGAGCACCCAGTCCGCCCTGA GCAAAGACCCCAACGAGAAGCGCGATCACATGGTCCTG CTGGAGTTCGTGACCGCCGCCGGGATCACTCTCGGCAT GGACGAGCTGTACAAGTAACATGCCCCAGAACTGCTGG GACCCGAATGT

\section{RHA}

CATGCCCCAGAACTGCTGGGACCCGAATGTTGGGTCC TTGAGGGTCACCTCTTTGGTCAAGAAAGGCATTCAGCT CTAACTGCTCCTTGATACCACGTGGCTTGGCCATTGCT GGTGCCAAGGCTGACCCCGAACTGGCAGAGCCGATGCC CTCTGGTGCACCCCAGGAAACATCTCCCCAAGTTCCAG CGCCCTTAATGACTCTTGCCACCCTGGGGGCTTCACCC TAACGCACCACTTCTCTGGAAGGGGAAGGCCAGACACA TGCCAGTTGGGGCTGCATGAGGCAGTCCTCAGAGCAGA AGGGGACCAGGCCAGAGGCCACCTGTGACGGGGCAAAC TGCATCTCGGCTGTGGAGACCAGAGGGGCTGTTAGATT TGGAAGACATCAATGACTGGGCCTGCGGCGCAGCCCGT GTCTGGTAATACCAGGGACGGCAGAGGCGTITGCATCT TCCCATCACCTGCAATGTCGCTGTCACTCTGCCCCTGT TCAGTGGACTTGATTAGTTAGGAAACTTCTGGAAGGGG CCCCCTACTTTATATCACAGAGTTTGCCCTAGACACCC CGTGGAAACACAAACTCAAAATCAAGTGGCTTTAGGAG CCGCTGTGCCCCTCCACAAGCTGACATGGCTACTCTAA GGGTTCCTGCTGGGCTGGCTTTGCTACGCTTTCCTCAA GCTGCTTTCTTATTACCAGGATGCCTCACAGCTACAAA GTCCAATCTCACAGCACCCGCACTGGAAAATACTGTTC TCCATTTTTTTTCCAAGGCACCCTGCTTTATGATTGGCT CAGAGTTGGAATATGGGATGCAGGGCGTCTGGCTCTCT CAAGTGTCAAGCAACCCTGGCTTCCGCATGTGGGGCGA GGGGAGTGAGCAGAACTCTTCTCTGTCAGTCCACGAAG TAGACTAGAAGCCAACGGGTGCCTGGGGGCAACTGACG GTCTGGATTCTGACGCCCAGCCTGGAGCAGGGGCCTGG CCCATCCTATGCTCACACAGTGGTCTGGCAGCCTGAGC CCACACAACTCCTCAGTCCTTGACAATGCTTAGGCTCT GTTCTGAGGGACTCCCCACACCTCCATTCAGGGCTCCC CGAGTCCTCAGCTCTTCGGATGTGGATATATGACACAC TAGCATAATAAATCTTGATCTGGCTTGAGTCTCTCTGG ATTATCTGCACAGGTCTGCAGGAATGGGGTGTTCATGG GCTGGGGGCAGCATTGGGGGGAGGGCATCAGCCAGGTC ACAGAGCTGAGACTGTGTGTGGGTGCTCACAGGTGTTG GACCCACGTACCTGAGGAGTCCTGGAGGTGGTGTGTCA AACGTACAGACACTITGCTGCCTGGCTCTGCAGCTGGT GCAGCCATGCCACAGTCACTTCTTTGTGCTGTGGGACA AAGCTGGGGCAAAGTCATGGCTGCCAATTCCCTACAGA
TGGGTGAGAACTGACTGAGCCTCAGGGCGATATCAAGC TTATCGATAATCAACCTCT

\section{Results}

\section{Generation and genetic confirmation of Tmem119-} EGFP and Tmem119-CreERT2 knock-in lines

Access to highly specific fluorescent reporter lines for imaging and Cre-driver lines for functional manipulation is critical in our quest for understanding the roles of microglia in health and disease. Previously generated lines efficiently target microglia, but also other cells of the monophagocytic system (Wieghofer and Prinz, 2016). Among a group of more recently discovered microgliaspecific genes, we chose targeting the Tmem119 gene to generate such mouse lines (Bennett et al., 2016). Specifically, we opted for a transgenic approach that harnesses the Tmem119 locus to drive expression, while preserving endogenous Tmem119 expression. We designed a CRISPR/Cas9 strategy to insert EGFP or CreERT2, preceded by ribosomeskipping peptide porcine teschovirus-1 polyprotein (P2A), into the stop codon of murine Tmem119 (Fig. 1A). Two founder lines were established for Tmem119-EGFP and 5 for Tmem119-CreERT2.

To determine whether the donor DNA was inserted correctly, a PCR-based approach was used with primers spanning each junction. For both of the lines generated, specific $5^{\prime}$ and $3^{\prime}$ junction PCR products were obtained, whereas no bands were observed with DNA from wildtype (WT) animals (Fig. 1B). This indicates specific insertion of the transgenes into the Tmem119 locus. To further examine scarless integration at the junctions, PCR products from the CreERT2 line were subject to Sanger sequencing covering the junctions. Both $5^{\prime}$ and $3^{\prime}$ sequencing traces show endogenous bases corresponding to the WT sequence in intron 1 and the $3^{\prime}$ UTR (Fig. 1C,D). Additional sequencing confirmed absence of mutations across the entire targeted allele in both lines. Together, these results confirm the generation of Tmem119-EGFP and Tmem119-CreERT2 lines.

Although the function of endogenous Tmem119 is currently unknown, both its temporally distinct pattern and high expression level suggest functional importance of this gene (Bennett et al., 2016). We thus designed a knock-in approach with the objective of leaving endogenous expression intact. To test whether Tmem119 expression is actually unaffected, we quantified Tmem119 transcript levels in the Tmem119-EGFP line by qPCR. Examination of Tmem119 expression in WT and Tmem119-EGFP ${ }^{+/-}$mice revealed that transcript levels are comparable between genotypes (Fig. 1E; WT median 0.9717, $n=4$, EGFP ${ }^{+/}$median 0.9306, $\left.n=6, p=0.5667^{\mathrm{a}}\right)$. We further examined TMEM119 protein expression using immunofluorescence and found no difference between WT and Tmem119-EGFP ${ }^{+/-}$knock-in mice (Fig. 1F,G; WT median 0.9835, $n=4$, EGFP ${ }^{+/-}$median 1.117, $n=4, p=0.20^{\mathrm{b}}$ ) Together, these data support the conclusion that Tmem119 knock-in mice were generated with precision.

To further test whether the genetic engineering could affect the properties of microglia, we examined basic morphologic features of reconstructed immunostained microglia in WT and Tmem119-EGFP ${ }^{+/-}$knock-in mice 
(Fig. $1 H, I)$. Using this approach, we determined that the cell body area (Fig. 1J; WT median $50.67 \mu \mathrm{m}^{2}$, EGFP ${ }^{+/-}$ median $46.82 \mu \mathrm{m}^{2}, n=12$ microglia from 4 mice per genotype, $p=0.3429^{\circ}$ ), number of processes (Fig. $1 K$; WT median 6.167, EGFP $^{+/-}$median 5.667, $n=12$ microglia from 4 mice per genotype, $p=0.4571^{d}$ ), convex hull area (Fig. 1L; WT median $2413 \mu \mathrm{m}^{2}$, $\mathrm{EGFP}^{+/-}$median $1871 \mu \mathrm{m}^{2}, n=12$ microglia from 4 mice per genotype, $p$ $=0.4857^{\circ}$ ), and total process length (Fig. $1 \mathrm{M}$; WT median $351.2 \mu \mathrm{m}, \mathrm{EGFP}^{+/-}$median $419.6 \mu \mathrm{m}, n=12$ microglia from 4 mice per genotype, $p=0.3428^{f}$ ) are comparable between microglia from control and knock-in mice. These data suggest that the genetic modification does not affect basic microglial properties.

\section{EGFP is faithfully expressed in microglia}

Microglia populate the parenchyma in virtually all regions across the brain. To determine the extent of EGFP expression in the newly generated Tmem119-EGFP line, we used confocal microscopy on brain slices. Native EGFP fluorescence and antibody-enhanced signal were visible across the brain in sagittal brain sections prepared from P25 Tmem119-EGFP ${ }^{+/-}$mice (Fig. 2A). To further assess whether EGFP is expressed in microglia, immunofluorescence labeling of the endogenous microglia proteins IBA1 and TMEM119 was used. High-power confocal microscopy shows that EGFP-positive cells in the parenchyma are IBA1- and TMEM119-positive (Fig. 2B-E). Completion and fidelity of expression were further assessed quantitatively in several brain regions. We found that $96.1 \pm 2.0 \%$ of the EGFP-expressing cells in the somatosensory cortex are IBA-1-expressing microglia and $98.2 \pm 3.3 \%$ of IBA1-positive cells in this region are labeled by EGFP (Table 1). In the striatum, $99.2 \pm 2.1 \%$ of the cells labeled with EGFP were IBA1-expressing and $97.2 \pm 1.8 \%$ of IBA1-positive cells displayed EGFP fluorescence. Labeling of microglia in the thalamus and hippocampus was $98.5 \pm 0.8 \%$ and $98.9 \pm 1.9 \%$ complete and $96.9 \pm 2.3 \%$ and $98.5 \pm 3.5 \%$ specific, respectively. To further confirm EGFP expression in microglia across the brain, we used the Tmem119-EGFP line in flow cytometry (Fig. $2 F-N$ ). We gated for single, live cells (Fig. $2 F-l$ ) and detected $\mathrm{EGFP}^{+}$cells among $\mathrm{CD}^{+} 5^{+}$cells (Fig. $J-M)$. We determined that all CD11 $\mathrm{b}^{+} \mathrm{CD} 45 \mathrm{lo}$ microglia (Fig. $2 K$ ) expressed EGFP (Fig. $2 N$ ). In addition, to more comprehensively assess specificity of EGFP expression, we immunostained oligodendrocytes, neurons, and astrocytes. We found no evidence for expression of EGFP in these major CNS cell types (Fig. 2O-Q). Together, these data indicate highly complete and specific labeling of parenchymal microglia in the Tmem119-EGFP reporter line.

All microglia express IBA1, but not all IBA1-expressing cells are parenchymal macrophages. In fact, nonparenchymal macrophages such as meningeal, perivascular, and choroid plexus macrophages, express the myeloid and macrophage marker IBA1 (Prinz and Priller, 2014). To determine whether EGFP is expressed in these non-parenchymal macrophages, we examined EGFP immunofluorescence in IBA1-expressing cells in the menin- ges and choroid plexus, which can be readily discerned by their morphology and IBA1 immunoreactivity. Highpower confocal images from both regions, including adjacent parenchyma, show that, whereas parenchymal microglia express the EGFP label, neither IBA1-positive meningeal (Fig. $3 A, B)$ nor choroid plexus macrophages express EGFP (Fig. 3C,D).

In contrast to the clear anatomic location of choroid plexus and meningeal macrophages, sites of perivascular macrophages are not easily discernible anatomically. To examine whether perivascular macrophages are labeled in the Tmem119-EGFP line, we stained for CD163, a well-characterized marker for perivascular macrophages (Kim et al., 2006). CD163 readily identified perivascular macrophages, which did not express EGFP (Fig. 3E,F). Adjacent microglia were clearly labeled with EGFP.

To further assess the specificity of EGFP expression in microglia beyond anatomic location-based immunofluorescence analysis, we used flow cytometry (Fig. 3G-l). Examining $\mathrm{CD} 45^{+} \mathrm{EGFP}^{+}$cells, we determined that 99.6\% are $\mathrm{CD} 11 \mathrm{~b}^{+} \mathrm{CD} 45$ lo cells corresponding to microglia (Fig. 3H). We further detected that 153 of 2212 CD11b ${ }^{+}$CD45int/hi cells $(6.9 \%)$ corresponding to nonmicroglial cells express EGFP (Fig. 3G-l). Together, these data indicate the capacity of the Tmem119-EGFP line to distinguish parenchymal microglia from other brain macrophages with good selectivity.

\section{EGFP is expressed in microglia of Tmem119-EGFP mice at early postnatal stages}

Microglia populate the brain during early embryonic development (Ginhoux et al., 2010). Tmem119 mRNA is expressed throughout microglial development, but antibody-based methods currently consistently label TMEM119 only as of later postnatal stages, typically P14 and later (Bennett et al., 2016). This makes it difficult to identify and discern microglia from monocytes at earlier time points. To determine whether the Tmem119-EGFP line would specifically label microglia at early postnatal stages, we conducted immunofluorescence microscopy at P1, P3, and P6. EGFP expression in IBA1-positive cells was readily observed in several regions examined, suggesting translation of Tmem119-P2A-EGFP transcript at this stage (Fig. 4A-L). Surprisingly, we also found EGFP expression associated with blood vessels of P1 mice (Fig. $4 A-C$ ), but not $\mathrm{P} 3$ or later, indicating that the transcript is also processed in cells of the intracerebral microvascular compartment at this stage. Together, these data indicate utility of the Tmem119-EGFP line for early developmental studies of microglia.

\section{Cre activity is specifically inducible in microglia of Tmem119-CreERT2 mice}

Cre activity in CreERT2 fusion protein is induced by the estrogen receptor antagonist tamoxifen and can be assessed in the presence of a reporter construct. To assess whether Tmem119-CreERT2 mice display Cre activity in parenchymal microglia on induction, we crossed Tmem119-CreERT2 mice to the Ai14 reporter line (Madisen et al., 2010), which expresses tdTomato in the presence of Cre (Fig. 5A). We administered tamoxifen 

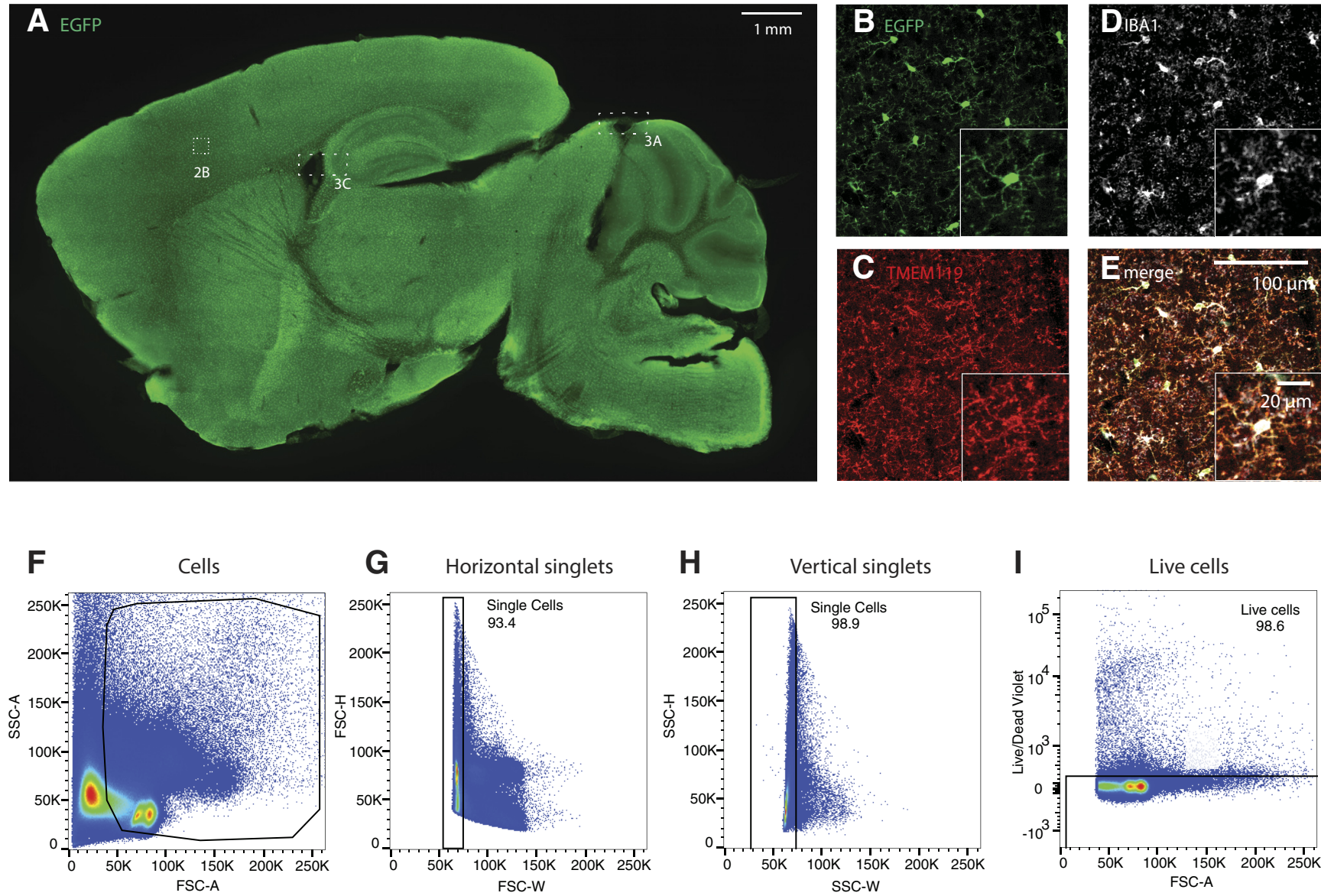

J CD45-positive myeloid cells
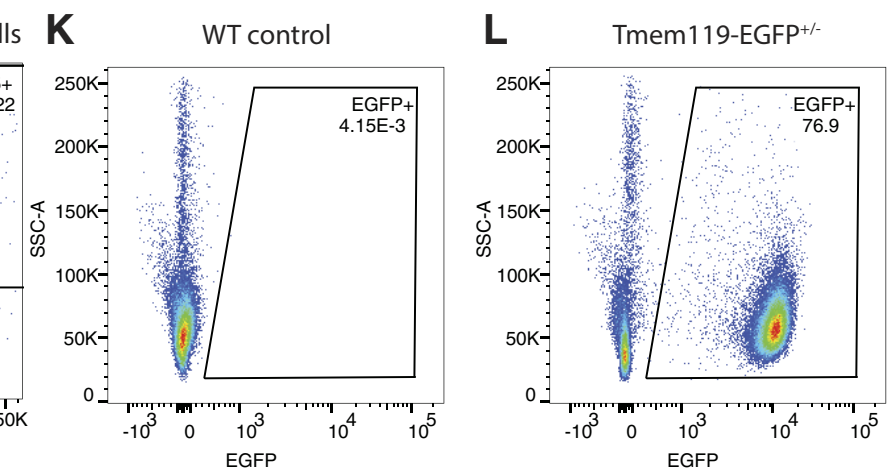

M CD11b+CD45lo microglia
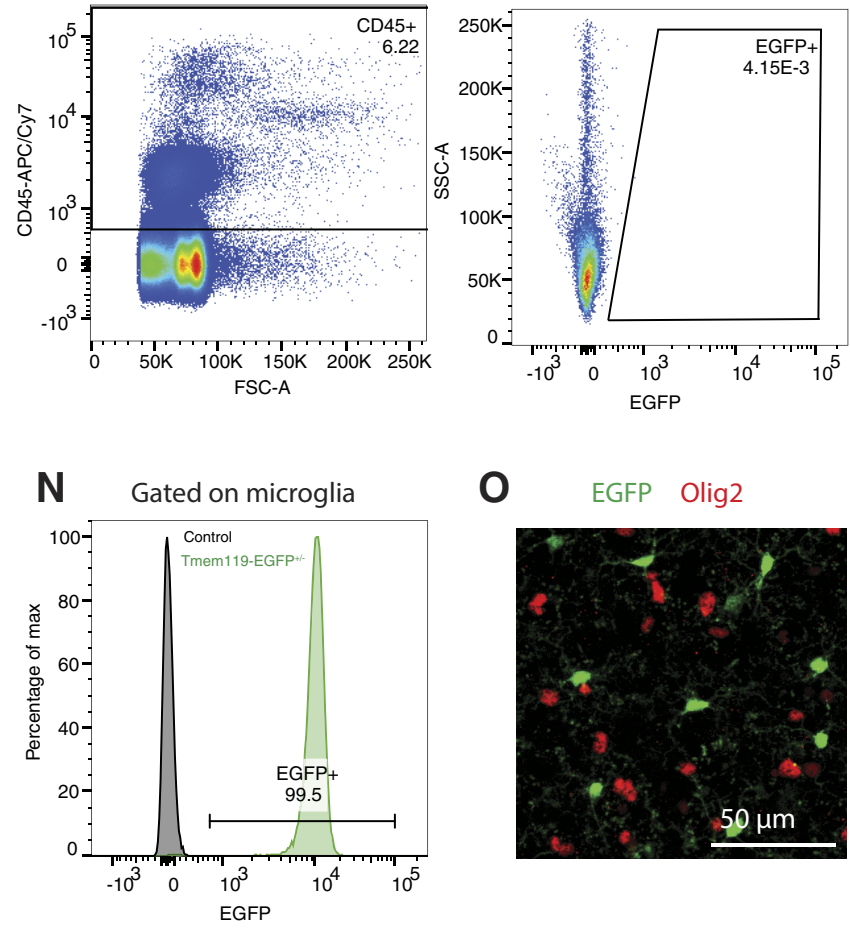

$\mathbf{P}$

EGFP NeuN

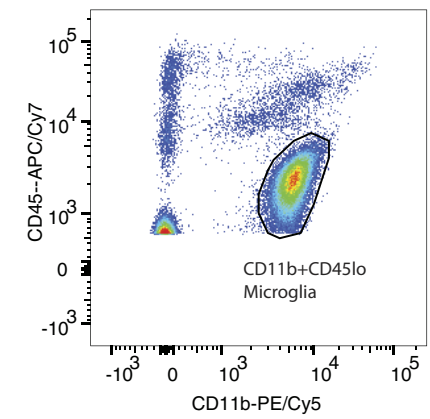

Q

EGFP

GFAP
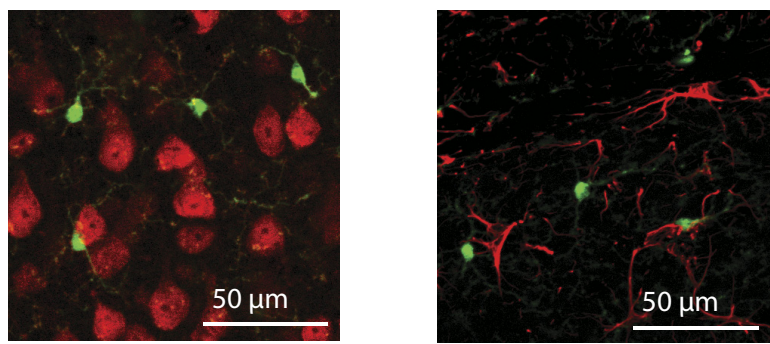

Figure 2. EGFP labels microglia in all regions across the brain. $\boldsymbol{A}$, Representative epifluorescence image of a sagittal section of a P25 Tmem119-EGFP ${ }^{+/-}$mouse stained with an anti-GFP antibody for improved signal-to-noise ratio (data for 1 of 3 mice shown). $\boldsymbol{B}-\boldsymbol{E}$, 


\section{continued}

High-power confocal micrographs of native EGFP fluorescence $(\boldsymbol{B}$, green) in TMEM119 (C, red) and IBA1 (D, white) microglia in the cortical region outlined in $\boldsymbol{A}$. FIJl-calculated composite image showing all three labels $(\boldsymbol{E})$. Boxes $3 \mathrm{~A}$ and $3 \mathrm{~B}$ Boxes cross-reference areas shown in Figure 3. $\boldsymbol{F}-\boldsymbol{J}$, Flow cytometry analysis showing gating for of single, live, CD45-positive cells (1 of 3 independent experiments shown). Numbers in or adjacent to outlined areas indicate percentage cells in each gate. $\boldsymbol{K}, \boldsymbol{L}$, Representative density plots showing EGFP expression in WT and Tmem119-EGFP ${ }^{+/-}$mice (pre-gated on CD45 ${ }^{+}$cells). $\boldsymbol{M}$, Representative density plot showing $\mathrm{CD}_{11 \mathrm{~b}}{ }^{+} \mathrm{CD} 45 \mathrm{lo}$ cells corresponding to microglia (pre-gated on $\mathrm{CD}_{4} 5^{+}$cells). $\boldsymbol{N}$, Histogram showing fraction of CD11 b ${ }^{+}$CD45lo microglia expressing EGFP. O-R, Representative immunostaining for oligodendrocytes $(\boldsymbol{O}$; Olig2, red), neurons $(\boldsymbol{P}$; NeuN, red), and astrocytes (Q; GFAP, red) in EGFP-expressing Tmem119-EGFP ${ }^{+/-}$mice. One of three independent experiments shown.

daily for 3,5 , or $10 \mathrm{~d}$ to adult mice and $3 \mathrm{~d}$ to $\mathrm{P} 2$ pups, and compared recombination efficiency and fidelity for these different paradigms (Fig. 5A). The mice were killed 9-10 d after the last dose and subjected to immunofluorescence staining. High-power images from confocal microscopy showed tdTomato-labeling of IBA1-expressing microglia in mice that received tamoxifen (Fig. $5 B-D$ ) and absence of tdTomato expression without tamoxifen (Fig. 5E-G). Confocal micrographs also indicated absence of tdTomato expression in other major cell types of the CNS, such as oligodendrocytes, neurons, and astrocytes (Fig. $5 H-J$ ). Assessment of the completeness of labeling as the ratio of tdTomato ${ }^{+} \mathrm{IBA} 1^{+}$double-positive microglia $\left(\mathrm{td} \mathrm{T}^{+} \mathrm{IBA} 1^{+}\right)$to all parenchymal microglia $\left(\mathrm{IBA} 1^{+}\right)$revealed very high efficiency of recombination (Fig. $5 K$ ). Similarly, we examined the fidelity of labeling which represents the fraction of actual parenchymal microglia $\left(\mathrm{tdT}^{+} \mathrm{IBA} 1^{+}\right)$ among all tdTomato-labeled cells $\left(\mathrm{tdT}^{+}\right)$in different parenchymal regions, and found high specificity close to $100 \%$ (Fig. $5 L$ ). Of note, increasing the days of tamoxifen administered to mice appeared to increase the fraction of microglia undergoing recombination. At the same time, prolonged administration of tamoxifen correlated with slightly reduced fidelity (Fig. $5 K, L$ ). We further sought to assess the specificity of tdTomato expression using flow cytometry. Among a total of $1690 \mathrm{CD} 11 \mathrm{~b}^{+} \mathrm{CD} 45 \mathrm{int} / \mathrm{hi}$ cells in the CNS, 33 were positive for tdTomato, indicating minimal, yet nonzero, expression in non-microglial myeloid cells.

The flow cytometry data revealed that recombination was largely, but not $100 \%$ specific. Furthermore, when examining the sections, we noticed some tdTomato fluorescence that did not appear to be associated with parenchymal microglia. To explore the nature of this ectopic fluorescence in this dosing paradigm, we imaged cells in the choroid plexus (Fig. $5 N-P$ ) as well as cells in the cortex, which were immunostained for the endothelial cell marker CD31 (Fig. 5Q-S). In the choroid plexus, there was very weak tdTomato fluorescence and most IBA1-positive

Table 1. Quantification of EGFP labeled microglia in Tmem119-EGFP mice

\begin{tabular}{lll}
\hline \multicolumn{1}{c}{ Brain region } & $\begin{array}{l}\text { Completeness, \% } \\
\left(\mathrm{EGFP}^{+} \mathrm{Iba}^{+} / \mathrm{Iba}^{+}\right)\end{array}$ & $\begin{array}{c}\text { Fidelity, \% } \\
\left(\mathrm{EGFP}^{+} \mathrm{Iba}^{+} / \mathrm{EGFP}^{+}\right)\end{array}$ \\
Somatosensory cortex & $98.2 \pm 3.3$ & $96.1 \pm 2.0$ \\
Striatum & $97.2 \pm 1.8$ & $99.2 \pm 2.1$ \\
Thalamus & $96.9 \pm 2.3$ & $98.5 \pm 0.8$ \\
Hippocampus & $98.5 \pm 3.5$ & $98.9 \pm 1.9$
\end{tabular}

macrophages did not express tdTomato (Fig. $5 N-P$ ). In the cortex, high-power confocal micrographs showed that the tdTomato and the CD31-immunolabeling signal were largely separate, with some tdTomato overlap with larger CD31-positive blood vessels (Fig. 5Q-S). Furthermore, tdTomato fluorescence was present on the pia in IBA1-negative cells (Fig. 5T- $V$ ) and absent from CD163expressing perivascular macrophages (Fig. 5W-Z). Together, these data indicate that the Tmem119-CreERT2 line can be used to conditionally control gene expression in adult and early postnatal microglia with good specificity.

\section{Characterization of transgene expression in monocytes from Tmem119 knock-in mice}

Monocytes infiltrate the brain and contribute to pathology in a number of brain disorders. Currently widely used transgenic microglia lines also target monocytes in addition to microglia and cannot readily distinguish the two cell types, thus hampering unequivocal attribution of microglia function in these disorders. To assess the utility of Tmem119 knock-in mice for investigating microglia in models of CNS disorders, we isolated blood monocytes and examined transgene expression (Fig. 6A). Using flow cytometry on white blood cells isolated from control or Tmem119-EGFP ${ }^{+/-}$mice, we did not detect any EGFPexpression (Fig. 6B,C). To further determine whether Tmem119-CreERT2 ${ }^{+/-}$; $\mathrm{Ail4}^{+/-}$mice display recombination in monocytes, we administered tamoxifen and killed the mice for analysis $7 \mathrm{~d}$ after the last dose (Fig. $6 D$ ). Using flow cytometry on white blood cells, we found that $\sim 3 \%$ of $\mathrm{CD} 45^{+}$cells expressed tdTomato (Fig. $6 E, F$ ). This suggests that there is a low level of CreERT2 activity in monocytes of the Tmem119-CreERT mice. Together, these data indicate high applicability of Tmem119-EGFP mice and good applicability of Tmem119-CreERT2 mice to the study of brain disorders with potential monocyte contribution.

\section{Discussion}

In this study, we addressed the critical need for transgenic mouse lines that specifically label or control microglia, but not other closely related cell types with overlapping signature gene expression (Bennett et al., 2016; Wieghofer and Prinz, 2016; Haimon et al., 2018). We targeted the recently identified microglia-specific Tmem119 gene to knock-in EGFP or CreERT2. Multiple analyses confirm correct insertion of the transgenes, intact expression of endogenous Tmem119, and normal 

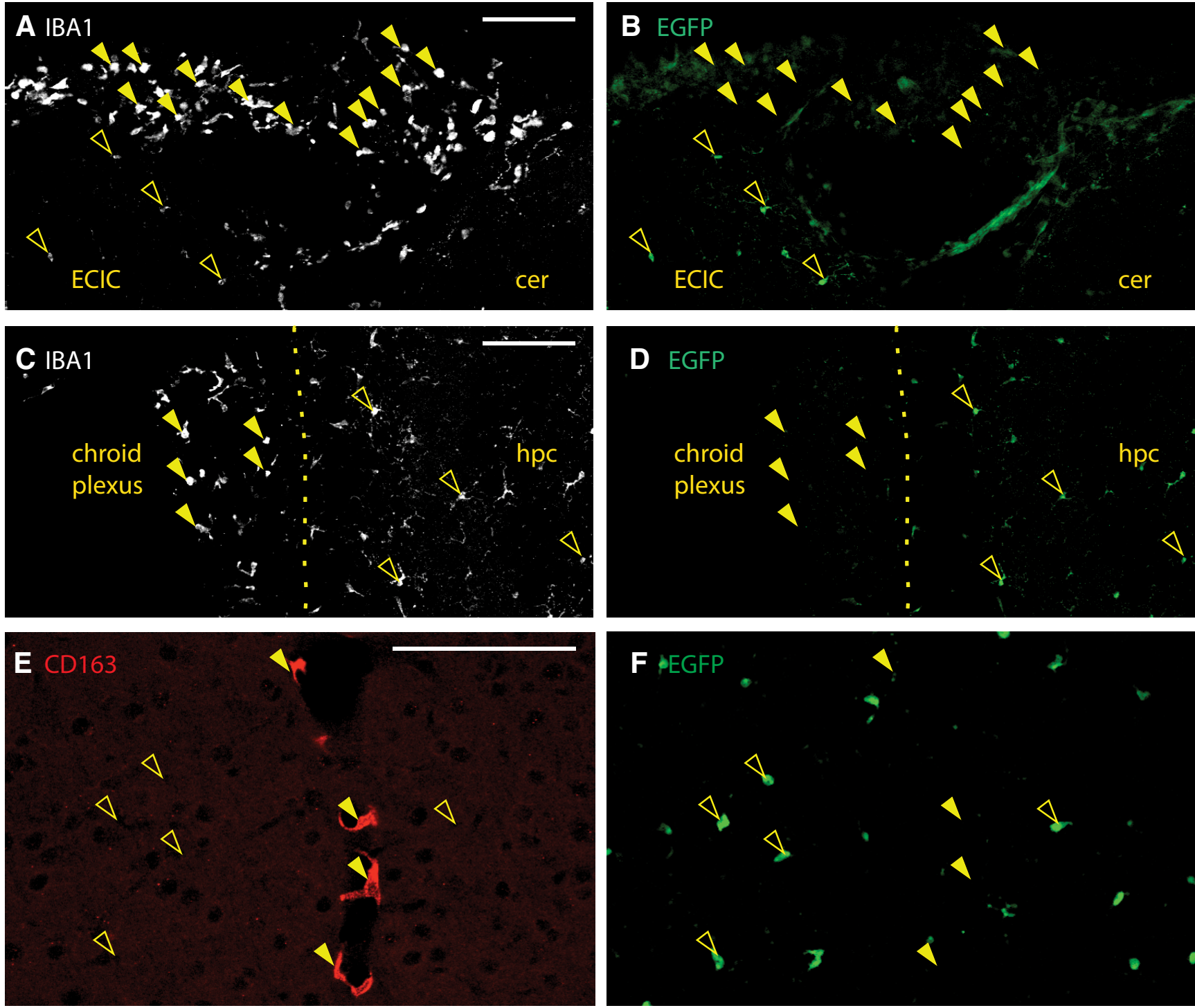

G

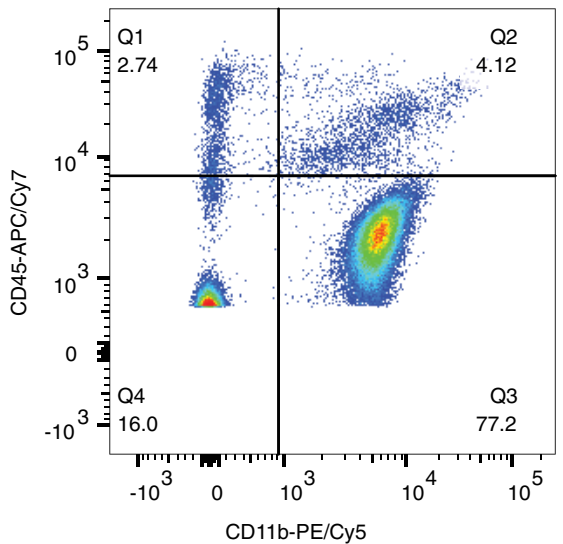

H $\quad \mathrm{CD}^{2} 5^{+} \mathrm{EGFP}^{+}$cells

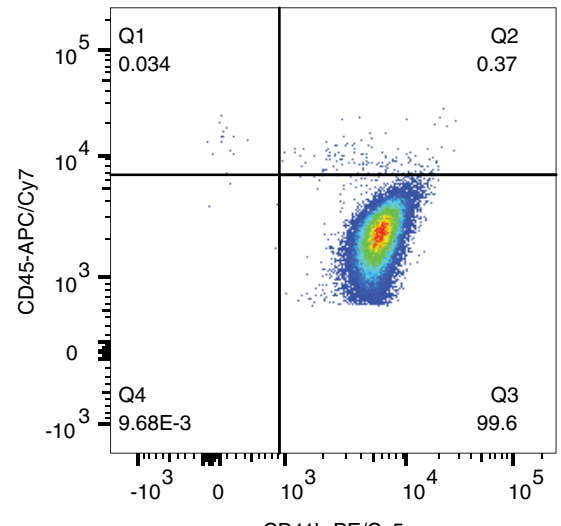

I Overlay

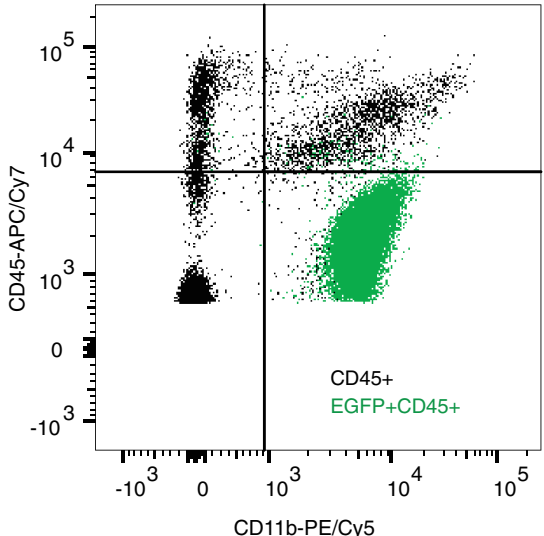

Figure 3. EGFP discerns parenchymal microglia from other brain macrophages in the Tmem119-EGFP line. A, IBA1 labels meningeal macrophages and parenchymal microglia from the region indicate with a dashed box in Figure $2 A$. $\boldsymbol{B}$, EGFP labels only parenchymal macrophages (open arrowheads), but not meningeal IBA1-positive macrophages (closed arrowheads). Diffuse fluorescence is associated with pia. ECIC, External cortex of inferior colliculus; Cer, cerebellum. Scale bar, $100 \mu \mathrm{m}$. C, $\boldsymbol{D}$, Choroid plexus IBA1 ${ }^{+}$ macrophages (closed arrowheads) from the region boxed in Figure $2 A$ are not labeled with EGFP, whereas adjacent parenchymal 
continued

microglia are (open arrowheads). Hpc, Hippocampus. Scale bar, $100 \mu \mathrm{m}$. $\boldsymbol{E}, \boldsymbol{F}$, Perivascular CD163-expressing macrophages (E, red, closed arrowheads) are not labeled with EGFP (F, green, open arrowheads). Scale bar, $100 \mu \mathrm{m}$. $\mathbf{G}-\boldsymbol{I}$, Flow cytometry analysis of CD11b and CD45 expression in pre-gated single live CD45 ${ }^{+}$cells and EGFP-expressing CD45 ${ }^{+}$cells of Tmem119-EGFP ${ }^{+/-}$mice shown as density plots $\left(\boldsymbol{G}, \mathrm{CD}_{4} 5^{+}\right.$and $\left.\boldsymbol{H} \mathrm{CD} 45^{+} \mathrm{EGFP}^{+}\right)$and overlay $\left(\boldsymbol{I}\right.$, black $\mathrm{CD} 45^{+}$, green $\left.\mathrm{CD} 45^{+} \mathrm{EGFP}^{+}\right)$. One of three independent experiments $(N \geq 3$ mice per group) shown. Numbers in or adjacent to outlined areas indicate percentage cells in each gate.

basic morphology of microglia in the knock-in mice. Using immunofluorescence and flow cytometry approaches, we demonstrate that EGFP is expressed in microglia, but not other cells of the monophagocytic system defined anatomically and by signature protein expression. Flow cytometry further shows that EGFP is almost exclusively expressed in CD11 ${ }^{+}$CD45lo cells corresponding to microglia. We further provide evidence for early postnatal expression of EGFP and activity of CreERT2 in microglia on tamoxifen administration. In addition, we show that blood monocytes do not express EGFP and display minimal activity of CreERT2. Together, our findings indicate proper generation of the two mouse lines, which are now readily available through a public repository (JAX\#031823 for Tmem119-EGFP and JAX\#031820 for Tmem119CreERT2).

TMEM119 is a single transmembrane protein that is highly expressed in microglia in the brains of humans and mice (Bennett et al., 2016; Satoh et al., 2016). In this study, we opted to refrain from knocking Tmem119 out through insertion of EGFP or CreERT2 into the Tmem119 ORF and instead chose to preserve expression by using a polycistronic knock-in approach with ribosomal skipping peptide P2A (Fig. 1A). This was primarily motivated by the high expression levels of Tmem119, conservation of expression in microglia across species, and potential developmental regulation patterns, all of which suggest the importance of this gene. Disruption of important genes in microglia could lead to confounding haploinsufficiency phenotypes, as previously shown for other transgenic microglia lines (Lee et al., 2010; Rogers et al., 2011). Our data rule out such potential confounding effects by showing intact Tmem119 mRNA and protein expression (Fig. $1 E-G)$, as well as normal basic morphology of microglia in the knock-in mice (Fig. $1 H-M$ ).

Examination of EGFP expression and co-expression analysis with TMEM119, IBA1, and CD163 revealed that EGFP labels only parenchymal microglia (Figs. 2, 3). Iden-
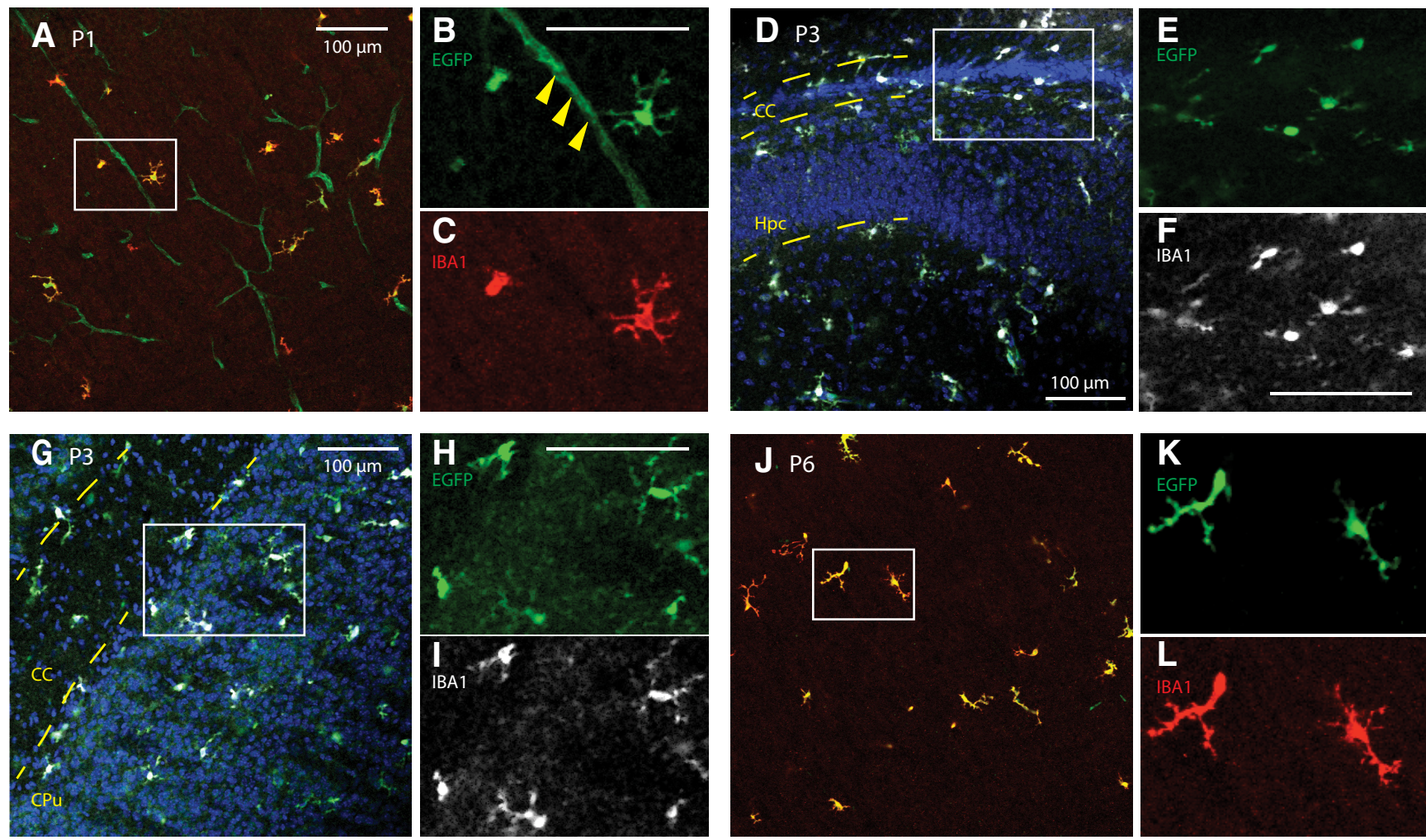

Figure 4. Tmem119-EGFP mice label microglia at early postnatal stages. Sagittal slices from P1, P3, and P6 mice were stained against IBA1. A-C, High-power confocal images of the somatosensory cortex show EGFP expression in IBA-positive cells and blood vessels at P1 (solid arrowheads in magnified panel). $\boldsymbol{D}-\boldsymbol{F}$, Confocal micrographs around the forceps major of corpus callosum (CC) and hippocampus (Hpc) show EGFP-labeled, IBA1-positive microglia at P3. G-I, Confocal micrographs of the striatum (CPu) and CC show EGFP labeling of IBA-positive cells at P3. $\boldsymbol{J}-\boldsymbol{L}$, Confocal micrographs of the P6 cortex reveal EGFP-expression in IBAimmunostained cells. Error bars, $100 \mu \mathrm{m}$. 
A
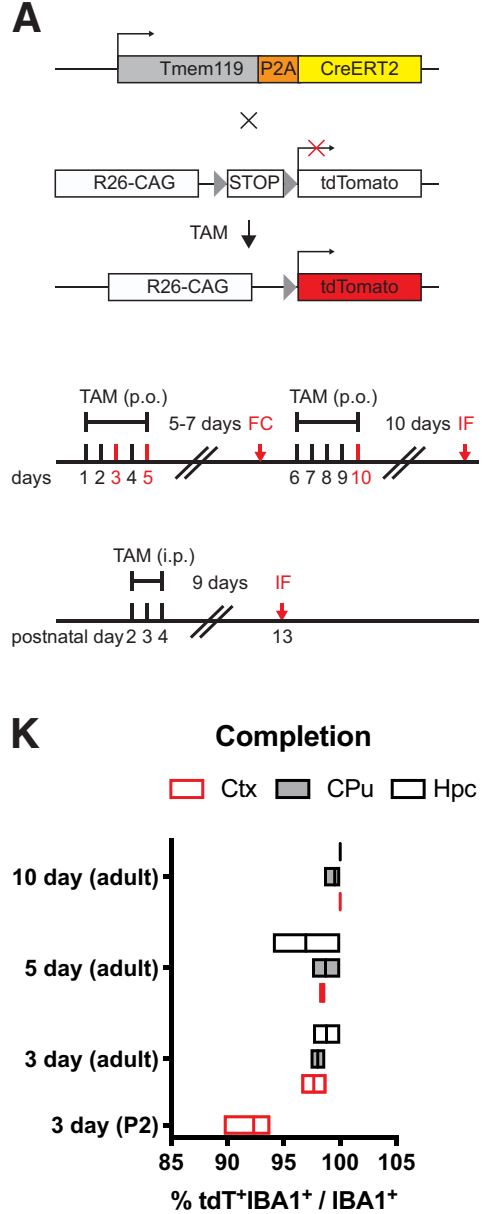

$\mathbf{N}$
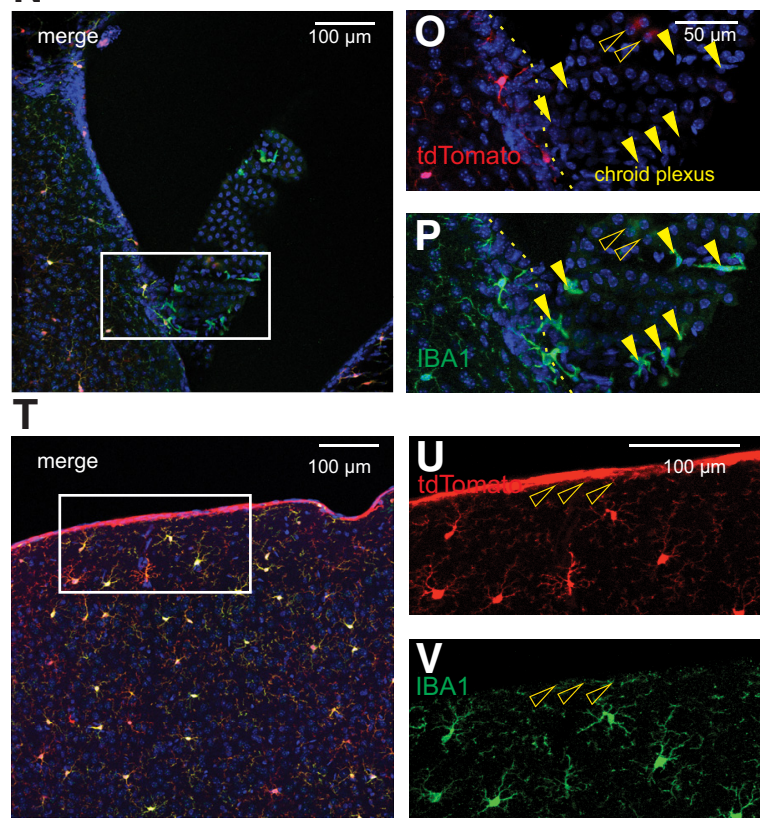

Figure 5. Tmem119-CreERT2 mice effectively recombine a conditional allele in microglia. $\boldsymbol{A}$, Tmem119-CreERT2 mice were crossed to $A i 14(R C L-t d T)-D$ mice and tamoxifen (TAM) was administered to induce tdTomato expression. Different sets of adult mice received tamoxifen per os (p.o.) for 3, 5, or $10 \mathrm{~d}$ and a set of neonatal mice received TAM for $3 \mathrm{~d}$ intraperitoneally (i.p). The mice were killed for flow cytometry (FC) $7 \mathrm{~d}$ after the $3 \mathrm{~d}$ dosing paradigm or for immunofluorescence (IF) 9 or more days after dosing. $\boldsymbol{B}$ - $\boldsymbol{D}$, Representative confocal micrograph showing tdTomato expression in IBA-positive microglia upon tamoxifen administration. $\boldsymbol{E}$-G, 
continued

Absence of tdTomato expression in untreated animals. $\boldsymbol{H} \boldsymbol{J}$, Representative immunostaining for oligodendrocytes $(\boldsymbol{H}$, Olig2, green), neurons (I, NeuN, green), and astrocytes (J, GFAP, green) in tdTomato-expressing microglia of Tmem119-CreERT2 ${ }^{+/-}$; Ai14(RCL$t d T)-D^{+/-}$mice. One of three independent experiments shown. $\boldsymbol{K}, \boldsymbol{L}$, Percentage completion $(\boldsymbol{K})$ and fidelity $(\boldsymbol{L})$ of the labeling in different brain regions for the different dosing schemes. Hpc, Hippocampus; CPu, caudate-putamen; Ctx, cortex. $N=2$ mice for 3 d, 3 mice each for 5 and $10 \mathrm{~d}$ of TAM in adults, and 3 mice for $3 \mathrm{~d}$ administration at P2. Floating bars = min to max, line at mean. $\boldsymbol{M}$, Flow cytometry analysis of CD11b and CD45 expression in pre-gated single live CD45 ${ }^{+}$cells and tdTomato-expressing CD45 ${ }^{+}$ cells of Tmem 119-CreERT2 ${ }^{+/-}$; Ai14(RCL-tdT)- $D^{+/-}$mice shown as overlay (I, black CD45 ${ }^{+}$, red CD45 ${ }^{+}$tdTomato $\left.^{+}\right)$. One of two representative experiments $(\boldsymbol{N}=3$ mice). $\mathbf{N}-\mathbf{O}$, Confocal micrograph of the choroid plexus and adjacent parenchyma. $\mathbf{Q}-\mathbf{S}$, Confocal micrograph of CD31-immunostained blood vessels (green) shows tdTomato ${ }^{+}$microglia (arrowheads) and tdTomato expression in a large blood vessel (open arrowhead). $\boldsymbol{T}-\boldsymbol{V}$, Confocal micrograph showing tdTomato expression in cortical microglia and cells of the pia (open arrowheads). $\boldsymbol{W}-\boldsymbol{Z}$, Confocal micrograph showing CD163- (white) and IBA1-expressing (green) macrophages. The open arrowhead indicates a tdTomato ${ }^{-} \mathrm{CD} 163^{+} \mathrm{IBA} 1^{+}$perivascular macrophage. Closed arrowheads parenchymal microglia. One of three independent experiments shown ( $N=3$ mice).

tification of these cells was possible by direct observation of EGFP fluorescence as well as by analysis of immunostaining-enhanced tissue sections, supporting the conclusion that EGFP expression levels are robust. Flow cytometry analysis further showed expression of EGFP in virtually all $\mathrm{CD}_{11} \mathrm{~b}^{+} \mathrm{CD} 45 \mathrm{lo}$ microglia (Fig. $2 \mathrm{~N}$ ) and a minor fraction of $6.9 \%(153 / 2212)$ of $C D 11 b^{+}$ CD45int/hi cells (Fig. 3G-I). This reflects that Tmem119 expression is highly selective, but not completely restricted to microglia, which has been observed in recent single-cell sequencing studies (Li et al., 2019). An interesting observation was the expression of EGFP at early
A

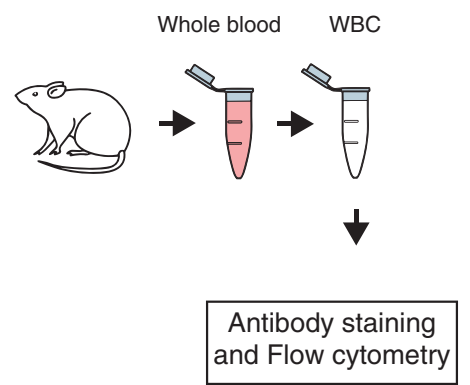

D
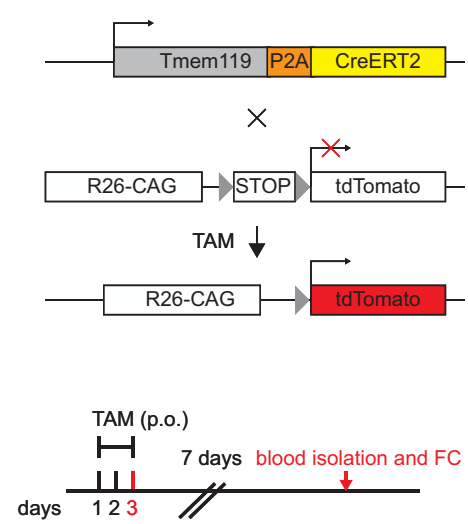

B

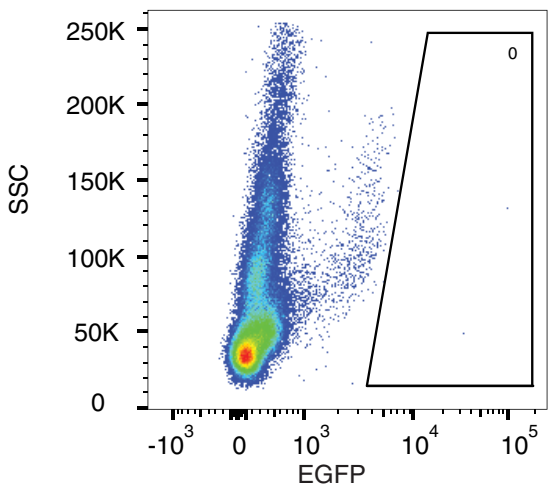

$\mathrm{Ai}^{+1 /-}$

E

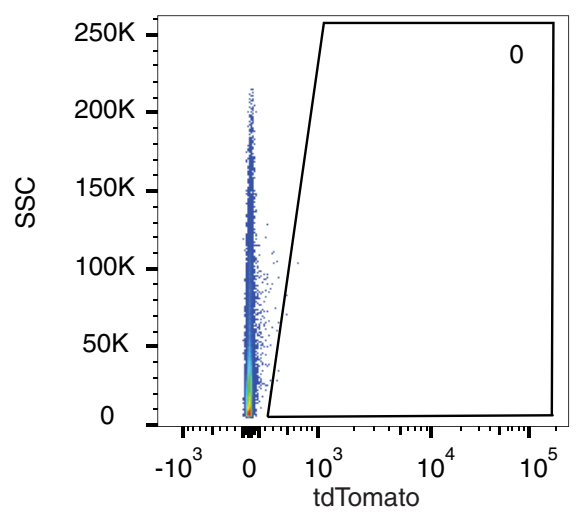

C Tmem119-EGFP+/- CD45+ myeloid cells

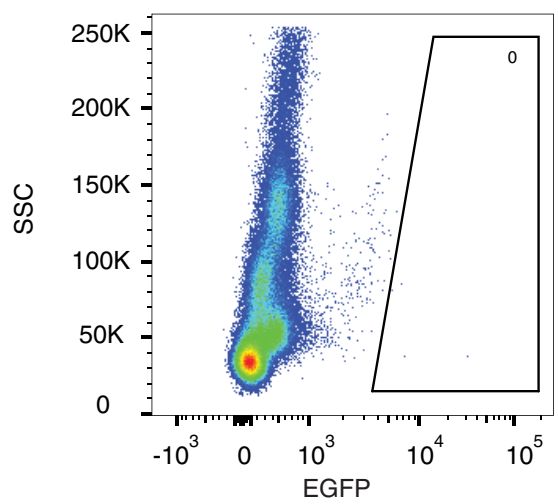

F

Tmem119-CreERT2 ${ }^{+/-}, \mathrm{Ai}_{14}{ }^{+/-}$ CD45+ myeloid cells

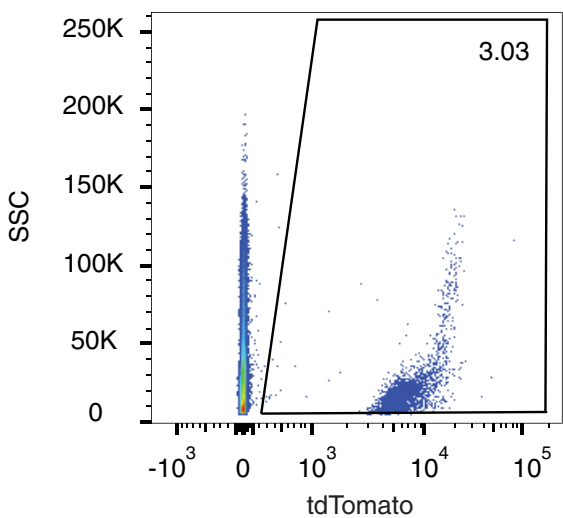

Figure 6. Blood monocyte profiling in Tmem119 knock-in mice. A, Experimental approach for flow cytometry of monocytes isolated from whole blood. $\boldsymbol{B}, \boldsymbol{C}$, Representative flow cytometry analysis of EGFP expression in pre-gated single live CD45 ${ }^{+}$cells in WT control (B) and Tmem119-EGFP ${ }^{+/-}$mice $(\boldsymbol{C})$. One of three experiments is shown ( $N=3$ mice). Numbers in or adjacent to outlined areas indicate percentage cells in each gate. $\boldsymbol{D}$, Adult mice of Tmem119-CreERT2 ${ }^{+/-}$; Ai14(RCL-tdT)- $D^{+/-}$mice received tamoxifen (TAM) per os (p.o.) for $3 \mathrm{~d}$. The mice were sacrificed $7 \mathrm{~d}$ after the last dose for flow cytometry (FC). $\boldsymbol{E}$, $\boldsymbol{F}$, Representative flow cytometry analysis of tdTomato expression in pre-gated single live CD45 ${ }^{+}$cells in Ai14(RCL-tdT)- $D^{+/-}$(E) and Tmem119-CreERT2 ${ }^{+/-}$; Ai14(RCL-tdT)- $D^{+\prime-}$ mice $(\boldsymbol{F})$. One of two independent experiments is shown $(N=3$ mice). 
Table 2. Statistical table

\begin{tabular}{llll}
\hline & \multicolumn{1}{c}{ Data structure } & \multicolumn{1}{c}{ Type of test } & 95\% Confidence interval of difference between medians \\
a & $N$ too small to test normality & Mann-Whitney test & -0.3393 to 0.2262 \\
b & $N$ too small to test normality & Mann-Whitney test & -0.1601 to 0.3359 \\
c & $N$ too small to test normality & Mann-Whitney test & -11.59 to 7.300 \\
d & $N$ too small to test normality & Mann-Whitney test & -2.000 to 1.000 \\
e & $N$ too small to test normality & Mann-Whitney test & -963.9 to 560.5 \\
f & $N$ too small to test normality & Mann-Whitney test & -109.0 to 120.0 \\
\hline
\end{tabular}

postnatal time points in microglia (P1, P3, and P6), but also endothelial cells of blood vessels at P1 (Fig. 4). This is interesting for two reasons. First, it suggests that Tmem119 is initially expressed in microglia and endothelial cells and only becomes a specific microglia marker during early postnatal development, presumably by selective upregulation and downregulation of expression in microglia and endothelial cells, respectively. Second, translational processing of Tmem119 transcript appears to stand in opposition to a previous report showing that, while RNA sequencing detects Tmem119 mRNA throughout development, immunostaining only identifies TMEM119 protein at later postnatal stages (Bennett et al., 2016). Although our data also do not directly show TMEM119 protein expression at early stages, ribosomal processing of the transcript is at least indirectly implied by the presence of EGFP protein, suggesting TMEM119 protein might be expressed but undetected because of expression level, protein stability, differential posttranslational modification, or methodological limitations in immunostaining and flow cytometry. Together, these data indicate that Tmem119 knock-in mice may enable the study of microglia and endothelial cells until P1 and microglia specifically after P3 and later.

Our experiments using Tmem119-CreERT2 ${ }^{+/-}$Ai14(RCL$\mathrm{tdT})^{+/-}$mice show tdTomato expression in IBA1expressing microglia, indicating that this line is suitable for Cre-dependent manipulation of genes and transgene expression in microglia without affecting other major neuronal or glial cell types in the CNS (Fig. 5). In this particular experiment, we observed very high, dose-dependent recombination efficiencies close to $100 \%$ with a trend for slight reduction of the generally high specificity with increasing tamoxifen dosage (Fig. $5 K, L$ ). The recombination efficiency observed across all doses compares well with other published models, such as the Cx3cr1 mouse line (Goldmann et al., 2013), and might even permit shorter dosing paradigms dependent on the assay and relevant conditional allele. We further examined tissues other than parenchymal microglia and observed that recombination was largely yet not completely absent in choroid plexus macrophages (Fig. $5 N-P$ ). This is consistent with reported endogenous TMEM119 expression and other reports in the literature, suggesting other brain macrophages may express microglia-specific genes at very low levels (Bennett et al., 2016; Goldmann et al., 2016; Mildner et al., 2017), which might be sufficient to cause occasional allor-none recombination events. In addition, our data showed tdTomato expression in a few larger blood vessels and IBA1-negative cells in the pia (Fig. 5Q-V). Although we did not determine the precise cellular origin of this signal, our data from CD31-immunostaining exclude endothelial cells of the intracerebral microvasculature
(Fig. 5Q-S). Together with the spatial extent of tdTomato expression and recent single-cell RNA sequencing data of vascular cells, our observations point to leptomeningeal cells and their projections that penetrate deep into the brain ensheathing large blood vessels and the choroid plexus stroma (Decimo et al., 2012, Vanlandewijck et al., 2018). Together, these data render the Tmem119CreERT2 line a highly useful tool for the conditional study of microglia, while also suggesting potential application for leptomeningeal cells.

Examination of EGFP and tdTomato expression in blood monocytes of Tmem119-EGFP and Tmem119-CreERT2; Ai14 mice revealed complete absence and minimal expression, respectively (Fig. 6). While, the lack of EGFP expression in blood monocytes of the Tmem119-EGFP knock-in mice was expected based on previous observations, tdTomato expression in 3\% blood monocytes of the Tmem119-CreERT2; Ai14 mice was rather surprising. Similar to other brain macrophages discussed above, blood monocytes may express Tmem119 at very low, almost undetectable levels, which may nonetheless be sufficient to cause occasional all-or-none Cre recombination events. Together, these data render the Tmem119 knock-in lines powerful tools to investigate microglia in health and disease without significant confounds from monocyte contribution.

During the past decade, available microglia lines have been instrumental in advancing our understanding of microglia. These lines remain powerful tools to study microglia function. At the same time, it is widely acknowledged that ontogenetic differences, as well as differences in localization and microenvironment between parenchymal microglia and their closely related cells from the monophagocytic system warrant more discerning observation and manipulation (Bennett et al., 2016; Wieghofer and Prinz, 2016; Haimon et al., 2018). Recently developed antibodies for TMEM119 immunostaining are one tool that has been adopted quickly to identify parenchymal microglia. As TMEM119 is a transmembrane protein localizing to processes, immunostaining for this protein is particularly powerful to study morphology of microglia. To identify discrete microglia, however, relatively diffuse staining patterns of membrane-bound TMEM119 present a challenge (Fig. 2C). In this case, the more discretely identifiable cytosolic distribution of EGFP in the Tmem119-EGFP line might be of great value (Fig. $2 B$ ). In addition, the robustness of the EGFP signal in the Tmem119-EGFP line may enable cell sorting without the need for antibodies, possibly even at early developmental stages before the onset of TMEM119 appearance on the membrane, as well as in vivo imaging approaches. Furthermore, the absence 
of EGFP fluorescence in blood monocytes renders this line uniquely useful for the study of microglia in brain disorders that involve monocyte infiltration. The Tmem119CreERT2 line will significantly facilitate conditional control of gene expression selectively in microglia, especially in cases when monocytes, but not leptomeningeal cells, could likely confound interpretations. Compared with constitutive Cre lines, which cumulatively recombine throughout development and thus deny temporal control, the CreERT2 line will allow precise control of gene expression to examine the role of microglia in temporally restricted developmental processes. In this capacity, dosedependency of CreERT2 and potentially resulting mosaic expression of recombined alleles using low doses may allow the study of candidate genes in adjacent microglia populations sharing the same microenvironment. Beyond the application of Tmem119 knock-in and other currently available mouse lines, genetic approaches such as targeting other microglia-signature genes, engineering highly specific artificial promoters and regulatory regions, and using intersectional split-Cre approaches hold great promise to produce even more specific tools to study microglia in the future.

\section{References}

Aguzzi A, Zhu C (2017) Microglia in prion diseases. J Clin Invest 127:3230-3239.

Bennett ML, Bennett FC, Liddelow SA, Ajami B, Zamanian JL, Fernhoff NB, Mulinyawe SB, Bohlen CJ, Adil A, Tucker A, Weissman IL, Chang EF, Li G, Grant GA, Hayden Gephart MG, Barres BA (2016) New tools for studying microglia in the mouse and human CNS. Proc Natl Acad Sci U S A 113:E1738-E1746.

Butovsky O, Jedrychowski MP, Moore CS, Cialic R, Lanser AJ, Gabriely G, Koeglsperger T, Dake B, Wu PM, Doykan CE, Fanek Z, Liu L, Chen Z, Rothstein JD, Ransohoff RM, Gygi SP, Antel JP, Weiner HL (2014) Identification of a unique TGF- $\beta$-dependent molecular and functional signature in microglia. Nat Neurosci 17: 131.

Buttgereit A, Lelios I, Yu X, Vrohlings M, Krakoski NR, Gautier EL, Nishinakamura R, Becher B, Greter M (2016) Sall1 is a transcriptional regulator defining microglia identity and function. Nat Immunol 17:1397-1406.

Clausen B, Burkhardt C, Reith W, Renkawitz R, Förster I (1999) Conditional gene targeting in macrophages and granulocytes using LysMcre mice. Transgenic Res 8:265-277.

Davalos D, Grutzendler J, Yang G, Kim JV, Zuo Y, Jung S, Littman DR, Dustin ML, Gan WB (2005) ATP mediates rapid microglial response to local brain injury in vivo. Nat Neurosci 8:752.

Decimo I, Fumagalli G, Berton V, Krampera M, Bifari F (2012) Meninges: from protective membrane to stem cell niche. Am J Stem Cells 1:92-105.

Ferron M, Vacher J (2005) Targeted expression of Cre recombinase in macrophages and osteoclasts in transgenic mice. Genesis 41: 138-145.

Ginhoux F, Greter M, Leboeuf M, Nandi S, See P, Gokhan S, Mehler MF, Conway SJ, Ng LG, Stanley ER (2010) Fate mapping analysis reveals that adult microglia derive from primitive macrophages. Science 330:841-845.

Goldmann T, Wieghofer P, Müller PF, Wolf Y, Varol D, Yona S, Brendecke SM, Kierdorf K, Staszewski O, Datta M, Luedde T, Heikenwalder M, Jung S, Prinz M (2013) A new type of microglia gene targeting shows TAK1 to be pivotal in CNS autoimmune inflammation. Nat Neurosci 16:1618.

Goldmann T, Wieghofer P, Jordão MJ, Prutek F, Hagemeyer N, Frenzel K, Amann L, Staszewski O, Kierdorf K, Krueger M, Locatelli G, Hochgerner H, Zeiser R, Epelman S, Geissmann F, Priller J,
Rossi FM, Bechmann I, Kerschensteiner M, Linnarsson S, et al. (2016) Origin, fate and dynamics of macrophages at central nervous system interfaces. Nat Immunol 17:797.

Haimon Z, Volaski A, Orthgiess J, Boura-Halfon S, Varol D, Shemer A, Yona S, Zuckerman B, David E, Chappell-Maor L, Bechmann I, Gericke M, Ulitsky I, Jung S (2018) Re-evaluating microglia expression profiles using RiboTag and cell isolation strategies. Nat Immunol 19:636-644.

Hirasawa T, Ohsawa K, Imai Y, Ondo Y, Akazawa C, Uchino S, Kohsaka S (2005) Visualization of microglia in living tissues using Iba1-EGFP transgenic mice. J Neurosci Res 81:357-362.

Itagaki S, McGeer P, Akiyama H, Zhu S, Selkoe D (1989) Relationship of microglia and astrocytes to amyloid deposits of Alzheimer disease. J Neuroimmunol 24:173-182.

Keren-Shaul H, Spinrad A, Weiner A, Matcovitch-Natan O, DvirSzternfeld R, Ulland TK, David E, Baruch K, Lara-Astaiso D, Toth B, Itzkovitz S, Colonna M, Schwartz M, Amit I (2017) A unique microglia type associated with restricting development of Alzheimer's disease. Cell 169:1276-1290.e17.

Kim WK, Alvarez X, Fisher J, Bronfin B, Westmoreland S, McLaurin J, Williams K (2006) CD163 identifies perivascular macrophages in normal and viral encephalitic brains and potential precursors to perivascular macrophages in blood. Am J Pathol 168:822-834.

Lawson LJ, Perry VH, Dri P, Gordon S (1990) Heterogeneity in the distribution and morphology of microglia in the normal adult mouse brain. Neuroscience 39:151-170.

Lee S, Varvel NH, Konerth ME, Xu G, Cardona AE, Ransohoff RM, Lamb BT (2010) CX3CR1 deficiency alters microglial activation and reduces beta-amyloid deposition in two Alzheimer's disease mouse models. Am J Pathol 177:2549-2562.

Li Q, Cheng Z, Zhou L, Darmanis S, Neff NF, Okamoto J, Gulati G, Bennett ML, Sun LO, Clarke LE, Marschallinger J, Yu G, Quake SR, Wyss-Coray T, Barres BA (2019) Developmental heterogeneity of microglia and brain myeloid cells revealed by deep single-cell RNA sequencing. Neuron 101:207-223.e10.

Madisen L, Zwingman TA, Sunkin SM, Oh SW, Zariwala HA, Gu H, $\mathrm{Ng}$ LL, Palmiter RD, Hawrylycz MJ, Jones AR, Lein ES, Zeng H (2010) A robust and high-throughput Cre reporting and characterization system for the whole mouse brain. Nat Neurosci 13:133140.

Mathys H, Adaikkan C, Gao F, Young JZ, Manet E, Hemberg M, De Jager PL, Ransohoff RM, Regev A, Tsai LH (2017) Temporal tracking of microglia activation in neurodegeneration at single-cell resolution. Cell Rep 21:366-380.

Mildner A, Huang H, Radke J, Stenzel W, Priller J (2017) P2Y12 receptor is expressed on human microglia under physiological conditions throughout development and is sensitive to neuroinflammatory diseases. Glia 65:375-387.

O'Loughlin E, Madore C, Lassmann H, Butovsky O (2018) Microglial phenotypes and functions in multiple sclerosis. Cold Spring Harb Perspect Med 8:a028993.

Paolicelli RC, Bolasco G, Pagani F, Maggi L, Scianni M, Panzanelli P, Giustetto M, Ferreira TA, Guiducci E, Dumas L, Ragozzino D, Gross CT (2011) Synaptic pruning by microglia is necessary for normal brain development. Science 333:1456-1458.

Parkhurst CN, Yang G, Ninan I, Savas JN, Yates JR, Lafaille JJ, Hempstead BL, Littman DR, Gan WB (2013) Microglia promote learning-dependent synapse formation through brain-derived neurotrophic factor. Cell 155:1596-1609.

Prinz M, Priller J (2014) Microglia and brain macrophages in the molecular age: from origin to neuropsychiatric disease. Nat Rev Neurosci 15:300.

Ransohoff RM (2016) How neuroinflammation contributes to neurodegeneration. Science 353:777-783.

Rogers JT, Morganti JM, Bachstetter AD, Hudson CE, Peters MM, Grimmig BA, Weeber EJ, Bickford PC, Gemma C (2011) CX3CR1 deficiency leads to impairment of hippocampal cognitive function and synaptic plasticity. J Neurosci 31:16241-16250. 
Samokhvalov IM, Samokhvalova NI, Nishikawa SI (2007) Cell tracing shows the contribution of the yolk sac to adult haematopoiesis. Nature 446:1056.

Sasmono RT, Oceandy D, Pollard JW, Tong W, Pavli P, Wainwright BJ, Ostrowski MC, Himes SR, Hume DA (2003) A macrophage colony-stimulating factor receptor-green fluorescent protein transgene is expressed throughout the mononuclear phagocyte system of the mouse. Blood 101:1155-1163.

Satoh JI, Kino Y, Asahina N, Takitani M, Miyoshi J, Ishida T, Saito Y (2016) TMEM119 marks a subset of microglia in the human brain. Neuropathology 36:39-49.

Schafer DP, Lehrman EK, Kautzman AG, Koyama R, Mardinly AR, Yamasaki R, Ransohoff RM, Greenberg ME, Barres BA, Stevens B (2012) Microglia sculpt postnatal neural circuits in an activity and complement-dependent manner. Neuron 74:691-705.

Sierra A, Encinas JM, Deudero JJ, Chancey JH, Enikolopov G, Overstreet-Wadiche LS, Tsirka SE, Maletic-Savatic M (2010) Microglia shape adult hippocampal neurogenesis through apoptosiscoupled phagocytosis. Cell Stem Cell 7:483-495.

Stevens B, Allen NJ, Vazquez LE, Howell GR, Christopherson KS, Nouri N, Micheva KD, Mehalow AK, Huberman AD, Stafford B, Sher A, Litke Alan M, Lambris JD, Smith SJ, John SWM, Barres BA
(2007) The classical complement cascade mediates CNS synapse elimination. Cell 131:1164-1178.

Ueno M, Fujita Y, Tanaka T, Nakamura Y, Kikuta J, Ishii M, Yamashita $\mathrm{T}$ (2013) Layer $V$ cortical neurons require microglial support for survival during postnatal development. Nat Neurosci 16:543-551.

Vanlandewijck M, He L, Mäe MA, Andrae J, Ando K, Del Gaudio F, Nahar K, Lebouvier T, Laviña B, Gouveia L, Sun Y, Raschperger E, Räsänen M, Zarb Y, Mochizuki N, Keller A, Lendahl U, Betsholtz C (2018) A molecular atlas of cell types and zonation in the brain vasculature. Nature 554:475.

Weinhard L, di Bartolomei G, Bolasco G, Machado P, Schieber NL, Neniskyte U, Exiga M, Vadisiute A, Raggioli A, Schertel A, Schwab Y, Gross CT (2018) Microglia remodel synapses by presynaptic trogocytosis and spine head filopodia induction. Nat Commun 9:1228.

Wieghofer P, Prinz M (2016) Genetic manipulation of microglia during brain development and disease. Biochimica et Biophysica Acta 1862:299-309.

Yona S, Kim KW, Wolf Y, Mildner A, Varol D, Breker M, Strauss-Ayali D, Viukov S, Guilliams M, Misharin A, Hume David A, Perlman H, Malissen B, Zelzer E, Jung S (2013) Fate mapping reveals origins and dynamics of monocytes and tissue macrophages under homeostasis. Immunity 38:79-91. 